\title{
On the relaxed area of the graph of discontinuous maps from the plane to the plane taking three values with no symmetry assumptions
}

\author{
Giovanni Bellettini ${ }^{1,2} \cdot$ Alaa Elshorbagy ${ }^{2,3} \cdot$ Maurizio Paolini $^{4} \cdot$ Riccardo Scala $^{5}$
}

Received: 7 January 2019 / Accepted: 25 June 2019 / Published online: 9 July 2019

(c) Fondazione Annali di Matematica Pura ed Applicata and Springer-Verlag GmbH Germany, part of Springer Nature 2019

\begin{abstract}
In this paper, we estimate from above the area of the graph of a singular map $u$ taking a disk to three vectors, the vertices of a triangle, and jumping along three $\mathcal{C}^{2}$-embedded curves that meet transversely at only one point of the disk. We show that the singular part of the relaxed area can be estimated from above by the solution of a Plateau-type problem involving three entangled nonparametric area-minimizing surfaces. The idea is to "fill the hole" in the graph of the singular map with a sequence of approximating smooth two-codimensional surfaces of graph-type, by imagining three minimal surfaces, placed vertically over the jump of $u$, coupled together via a triple point in the target triangle. Such a construction depends on the choice of a target triple point, and on a connection passing through it, which dictate the boundary condition for the three minimal surfaces. We show that the singular part of the relaxed area of $u$ cannot be larger than what we obtain by minimizing over all possible target triple points and all corresponding connections.
\end{abstract}

Riccardo Scala

scala@mat.uniroma1.it

Giovanni Bellettini bellettini@diism.unisi.it

Alaa Elshorbagy

alaa.elshorbagy@sissa.it

Maurizio Paolini paolini@dmf.unicatt.it

1 Dipartimento di Ingegneria dell'Informazione e Scienze Matematiche, Università di Siena, 53100 Siena, Italy

2 Mathematics Section, International Centre for Theoretical Physics (ICTP), 34151 Trieste, Italy

3 Area of Mathematical Analysis, Modelling, and Applications, Scuola Internazionale Superiore di Studi Avanzati "SISSA", Via Bonomea, 265, 34136 Trieste, Italy

4 Dipartimento di Matematica e Fisica, Università Cattolica del Sacro Cuore, 25121 Brescia, Italy

5 Dipartimento di Matematica "Guido Castelnuovo", Università La Sapienza, Piazzale Aldo Moro 5, 00185 Rome, Italy 
Keywords Relaxation · Cartesian currents · Area functional · Minimal surfaces · Plateau problem

Mathematics Subject Classification 49Q15 · 49Q20 · 49J45

\section{Introduction}

Let $\Omega \subset \mathbb{R}^{2}$ be an open set and $v=\left(v_{1}, v_{2}\right): \Omega \rightarrow \mathbb{R}^{2}$ a Lipschitz map. It is well known that the area of the graph of $v$ is given by

$$
\mathbb{A}(v, \Omega)=\int_{\Omega} \sqrt{1+\left|\nabla v_{1}\right|^{2}+\left|\nabla v_{2}\right|^{2}+\left(\frac{\partial v_{1}}{\partial x} \frac{\partial v_{2}}{\partial y}-\frac{\partial v_{1}}{\partial y} \frac{\partial v_{2}}{\partial x}\right)^{2}} \mathrm{~d} x \mathrm{~d} y .
$$

Extending to nonsmooth maps via relaxation the definition of the area is a difficult question [10] and is motivated by rather natural problems in calculus of variations: we can mention for example the use of direct methods to face the two-codimensional Plateau problem in $\mathbb{R}^{4}$ in cartesian form, and the study of lower semicontinuous envelopes of polyconvex functionals with nonstandard growth [1,9]. A crucial issue is to decide which topology one has to consider in order to compute the relaxed functional of $\mathbb{A}(\cdot, \Omega)$ : of course, the weakest the topology, the most difficult should be the computation of the relaxed functional, but the easiest becomes the coerciveness. We recall that when $v$ is scalar valued, the natural choice is the $L^{1}(\Omega)$-convergence, and the relaxation problem is completely solved [3,7]; the $L^{1}(\Omega)$ relaxed functional in this case consists, besides the absolutely continuous part, of a singular part which is the total variation of the jump and Cantor parts of the distributional derivative of $v$ in $\Omega$; in particular, the relaxed functional, when considered as a function of $\Omega$, is a measure.

The case of interest here, namely when $v$ takes values in $\mathbb{R}^{2}$, is much more involved, due to the nonconvexity of the integrand in (1.1), and to the unilateral linear growth

$$
\mathbb{A}(v, \Omega) \geq \int_{\Omega} \sqrt{\left|\nabla v_{1}\right|^{2}+\left|\nabla v_{2}\right|^{2}} \mathrm{~d} x \mathrm{~d} y .
$$

Choosing again the $L^{1}\left(\Omega ; \mathbb{R}^{2}\right)$-convergence (as we shall do in this paper), the relaxed functional $\mathcal{A}(\cdot, \Omega)$ of $\mathbb{A}(\cdot, \Omega)$, i.e.,

$$
\mathcal{A}(v, \Omega):=\inf \left\{\liminf _{\epsilon \rightarrow 0} \mathbb{A}\left(u^{\epsilon}, \Omega\right):\left\{u^{\epsilon}\right\} \subset \operatorname{Lip}\left(\Omega ; \mathbb{R}^{2}\right), u^{\epsilon} \rightarrow u \text { in } L^{1}\left(\Omega ; \mathbb{R}^{2}\right)\right\},
$$

is, for $v \in L^{1}\left(\Omega ; \mathbb{R}^{2}\right) \backslash W^{1,2}\left(\Omega ; \mathbb{R}^{2}\right)$, far from being understood, and exhibits surprising features. One of the few known facts that must be pointed out is that, for a large class of nonsmooth maps $v$, the function $\Omega \rightarrow \mathcal{A}(v, \Omega)$ cannot be written as an integral [1,5,6]; this interesting phenomenon, related to nonlocality, has at least two sources. For simplicity, let us focus our attention on nonsmooth functions with jumps, thus neglecting the case of vortices. The first source of nonlocality has been enlightened answering to a conjecture in [8]. Specifically, consider the symmetric triple junction map $u_{\text {symm }}$, i.e., the singular map from a disk $D$ of $\mathbb{R}_{S}^{2}=\mathbb{R}^{2}$ into $\mathbb{R}_{T}^{2}=\mathbb{R}^{2}$, taking only three values-the vertices of an equilateral triangle $T_{\text {eq }} \subset \mathbb{R}_{T}^{2}$-and jumping along three segments meeting at the origin in a triple junction at equal $120^{\circ}$ angles: then $\mathcal{A}\left(u_{\text {symm }}, \cdot\right)$ is not subadditive. This result has been proven in [1]; subsequently in [4] it is shown that the value $\mathcal{A}\left(u_{\text {symm }}, D\right)$ is related to the solution of three one-codimensional Plateau-type problems in cartesian form suitably entangled together through the Steiner point in the triangle $T_{\mathrm{eq}}$. Due to the special symmetry of 


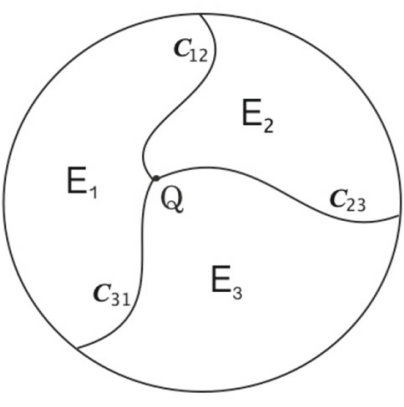

(a)

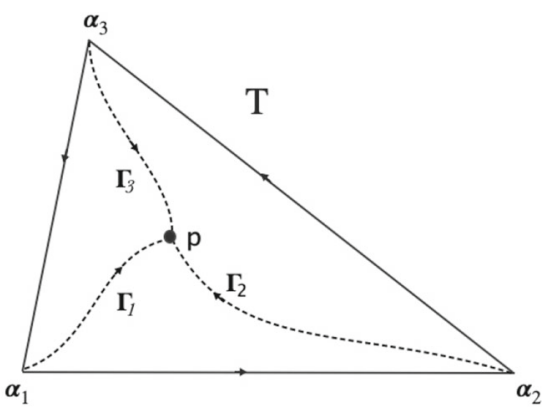

(b)

Fig. 1 a The domain of $u ; u=\alpha_{i}$ on $E_{i}$ and $\mathbf{b}$ a Lipschitz graph-type connection in the target triangle T. $\Gamma_{1} \cup \Gamma_{2}$ (resp. $\Gamma_{2} \cup \Gamma_{3}, \Gamma_{3} \cup \Gamma_{1}$ ) is graph over the segment $\overline{\alpha_{1} \alpha_{2}}$ (resp. $\overline{\alpha_{2} \alpha_{3}}, \overline{\alpha_{3} \alpha_{1}}$ ) of a Lipschitz function $\varphi_{12}\left(\right.$ resp. $\left.\varphi_{23}, \varphi_{31}\right)$

the map $u_{\text {symm }}$, the three problems collapse together to only one one-codimensional Plateautype problem in cartesian form, on a fixed rectangle $R$ whose sides are the radius of $D$ and the side of $T_{\text {eq }}$. Positioning three copies of this minimal surface "vertically" (in the space of graphs, i.e., in $D \times \mathbb{R}^{2}$ ) over the jump of $u_{\text {symm }}$ allows, in turn, to construct a sequence $\left\{u_{\varepsilon}\right\}$ of Lipschitz maps from $D$ into $\mathbb{R}^{2}$ the limit area of which improves the upper estimate of [1]. Optimality of this construction has been shown in the recent paper [12], on the basis of a symmetrization procedure for currents.

It is one of the aims of the present paper to inspect solutions of the above-mentioned three Plateau-type problems in more general situations, in order to provide upper estimates for $\mathcal{A}(u, D)$, for suitable piecewise constant maps $u$.

A second source of nonlocality for the functional $\mathcal{A}(u, \Omega)$ is given by the interaction of the jump set of a discontinuous map $u$ with the boundary of the domain $\Omega$. This phenomenon, already observed in [1] for the map with one-vortex at the center of a suitable disk, appears also for functions with jump discontinuities not piecewise constant [6]. More surprisingly, it appears also for piecewise constant maps taking three values, provided the jump is sufficiently close to the boundary of $\Omega$, as observed in [12], taking as $\Omega$ a sufficiently thin tubular neighborhood of the jump itself. We shall not be concerned here with this second source of nonlocality.

As already mentioned above, in this paper we are interested in estimating from above the area of the graph of a singular map $u$ taking three (non collinear) values and jumping along three embedded curves of class $\mathcal{C}^{2}$ that meet transversely at only one point, see Fig. 1. Let us state this in a more precise way, referring to Sects. 2 and 3 for all details. For simplicity, from now on we fix $\Omega$ to be an open disk $D$ containing the origin $0_{S}$ in the source plane $\mathbb{R}^{2}=$ $\mathbb{R}_{x, y}^{2}=\mathbb{R}_{S}^{2}$. Take three non-overlapping non-empty two-dimensional connected regions $E_{1}, E_{2}, E_{3}$ of $D$ such that

$$
E_{1} \cup E_{2} \cup E_{3}=D \text {. }
$$

The three regions are separated by three embedded curves of class $\mathcal{C}^{2}$ (up to the boundary) of length $r_{12}, r_{23}, r_{31}$, respectively, that meet only at $Q$ (source triple junction); moreover, each curve is supposed to meet the boundary of $D$ transversely and we assume also that $Q$ is a transversal intersection for the three curves, see Fig. 1a. 
Let $\alpha_{1}, \alpha_{2}, \alpha_{3}$ be the vertices of a closed triangle $\mathrm{T}$ with non-empty interior in the target plane.

Set

$$
\ell_{12}:=\left|\alpha_{1}-\alpha_{2}\right|, \quad \ell_{23}:=\left|\alpha_{2}-\alpha_{3}\right|, \quad \ell_{31}:=\left|\alpha_{1}-\alpha_{3}\right| .
$$

We suppose that $\mathrm{T}$ contains the origin $0_{\mathrm{T}}$ in its interior.

Let us introduce the space $X$ of connections (Definition 3.1 and (3.3), (3.4)); a connection $\Gamma=\left(\Gamma_{1}, \Gamma_{2}, \Gamma_{3}\right)$ consists of three rectifiable curves in $\mathrm{T}$, that connect the vertices of $\mathrm{T}$ to some point inside the triangle (called target triple point). We shall suppose that each curve can be written as a graph, possibly with vertical parts, over the corresponding two sides of T. When $\Gamma$ consists of three Lipschitz graphs, we write $\Gamma \in X_{\mathrm{Lip}}$, and we say that $\Gamma$ is a Lipschitz connection. We now show how to construct a new functional $\mathcal{G}$, consisting of the sum of the areas of three minimal surfaces - graphs of three suitable area-minimizing functions $m_{12}, m_{23}, m_{31}$ defined on certain rectangles-coupled together by the connection considered as a Dirichlet boundary condition, see Definition 3.4.

Set

$$
\mathrm{R}_{i j}:=\left[0, \ell_{i j}\right] \times\left[0, r_{i j}\right], \quad i j \in\{12,23,31\} .
$$

Assume $\Gamma \in X$. Then $\Gamma_{i j}:=\Gamma_{i} \cup \Gamma_{j}, i j \in\{12,23,31\}$ are (generalized) graphs of functions $\varphi_{i j}$ of bounded variation over $\left[0, \ell_{i j}\right]$. With a small abuse of notation, set

$$
\varphi_{i j}(s, t)=\varphi_{i j}(s), \quad(s, t) \in \mathrm{R}_{i j}, \quad i j \in\{12,23,31\} .
$$

The graph of $\varphi_{12}$ on $\mathrm{R}_{12}$ is depicted in Fig. 2a.

Let $m_{i j}=m_{i j}(\Gamma)$ be the unique solution of the Dirichlet-Neumann minimum problem, discussed in Sect. 3.1,

$$
\min \left\{\int_{\mathrm{R}_{i j}} \sqrt{1+|\nabla f|^{2}} \mathrm{~d} s \mathrm{~d} t: f \in W^{1,1}\left(\mathrm{R}_{i j}\right), f=\varphi_{i j} \mathcal{H}^{1}-\text { a.e. on } \partial_{D} \mathrm{R}_{i j}\right\},
$$

where

$$
\partial_{D} \mathrm{R}_{i j}=\partial \mathrm{R}_{i j} \backslash\left(\left[0, \ell_{i j}\right] \times\left\{r_{i j}\right\}\right), \quad i j \in\{12,23,31\} .
$$

Notice that the minimization is taken among all functions having a Dirichlet condition on three of the four sides of the rectangle $\mathrm{R}_{i j}$; the missing side corresponds to the intersection points of the jump with the boundary of $D$.

From (1.6) it follows that the Dirichlet condition is zero on the sides $\{0\} \times\left[0, r_{i j}\right]$ and $\left\{\ell_{i j}\right\} \times\left[0, r_{i j}\right]$ of $\mathrm{R}_{i j}$; see Fig. $2 \mathrm{~b}$.

Set

$$
\mathfrak{A}_{i j}(\Gamma):=\int_{\mathrm{R}_{i j}} \sqrt{1+\left|\nabla m_{i j}\right|^{2}} \mathrm{~d} s \mathrm{~d} t, \quad i j \in\{12,23,31\} .
$$

The main result of the present paper reads as follows (see Theorem 4.1 and Corollary 5.8).

Theorem 1.1 Let $u: D \rightarrow\left\{\alpha_{1}, \alpha_{2}, \alpha_{3}\right\}$ be the discontinuous $B V\left(D ; \mathbb{R}^{2}\right)$ function defined as

$$
u(x, y):= \begin{cases}\alpha_{1} & \text { if }(x, y) \in E_{1} \\ \alpha_{2} & \text { if }(x, y) \in E_{2} \\ \alpha_{3} & \text { if }(x, y) \in E_{3} .\end{cases}
$$




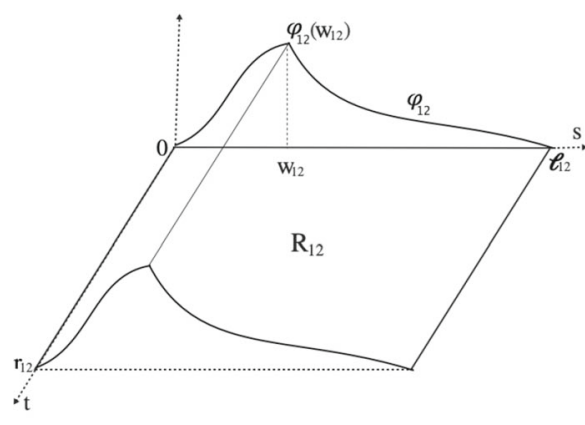

(a)

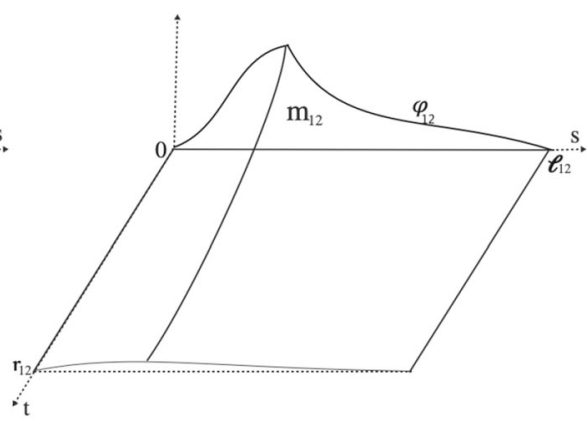

(b)

Fig. 2 a The graph of the function $\varphi_{12}$ on $\mathrm{R}_{12}$ and $\mathbf{b}$ the graph of $m_{12}$ on $\mathrm{R}_{12}$

Then

$$
\mathcal{A}(u, D) \leq|D|+\min \left\{\mathfrak{A}_{12}(\Gamma)+\mathfrak{A}_{23}(\Gamma)+\mathfrak{A}_{31}(\Gamma): \Gamma \in X\right\} .
$$

This theorem says that the singular part of $\mathcal{A}(u, D)$ can be estimated from above by

$$
\inf \left\{\mathfrak{A}_{12}(\Gamma)+\mathfrak{A}_{23}(\Gamma)+\mathfrak{A}_{31}(\Gamma): \Gamma \in X\right\},
$$

and that such an infimum is a minimum. Intuitively, to "fill the hole" in the graph of $u$ with smooth two-codimensional approximating surfaces of graph-type, we start to imagine three area-minimizing surfaces, placed vertically over the jump of $u$, coupled together via a triple point in the target triangle $\mathrm{T}$ (notice that the union of these three surfaces, viewed in $D \times \mathbb{R}^{2}$, is not smooth in correspondence of the source triple junction). Such a construction depends on the choice of a target triple point, and on a connection $\Gamma$ passing through it, dictating the boundary condition for the three area-minimizing surfaces, over the sides of the triangle T. Theorem 1.1 asserts that the interesting part of the relaxed area of $u$, namely its singular part, cannot be larger than what we obtain by minimizing over all possible target triple points and all corresponding connections. As a direct consequence of the results in $[4,12]$, when $u=u_{\text {symm }}$ (and $0_{S}$ is the center of $D$ ), the inequality in (1.10) is an equality, and the infimum in (1.11) is achieved by the Steiner graph connecting the three vertices of $\mathrm{T}$ (the optimal triple point being the Steiner point, i.e., the barycenter of T). This seems to be an interesting result that could be stated purely as a problem of three entangled area-minimizing surfaces (each of which lies in a half-space of $\mathbb{R}^{4}$, the three half-spaces having only $\{0\} \times \mathbb{R}^{2}$ in common) without referring to the relaxation of the functional $\mathbb{A}(\cdot, D)$. We do not know whether, in general, the Steiner graph is still the solution of the minimization problem in (1.11), when no symmetry assumptions (the case we are considering here) are required. However, it is reasonable to expect that, if in the source we have symmetry, i.e., the source triple junction is positioned at the center of $D$ and $u$ jumps along three segments meeting at equal $120^{\circ}$ angles, and if the target triangle $\mathrm{T}$ is close to be equilateral, the inequality in (1.10) to be still an equality. In this respect, it is worthwhile to observe that showing a lower estimate, for instance showing that, in certain cases, the inequality in (1.10) is an equality, seems difficult. One of the main technical obstructions is due to the poor control on the tangential derivative of $v^{\varepsilon}$ in proximity of the jump of a discontinuous $L^{1}$-limit function $v$ (see [6]), where $\left\{v^{\varepsilon}\right\}$ is a sequence of Lipschitz maps converging in $L^{1}\left(\Omega ; \mathbb{R}^{2}\right)$ to $v$, and satisfying the uniform bound $\sup _{\varepsilon} \mathbb{A}\left(v^{\varepsilon}, \Omega\right)<+\infty$. We also notice that the symmetrization methods of [12] cannot be applied anymore, in view of the lackness of symmetry. 
It is worth mentioning that the restriction that we assume on the connections $\Gamma$, namely that each $\Gamma_{i}$ is a graph (possibly with vertical parts) on the corresponding two sides of $\mathrm{T}$, cannot be avoided in our approach: indeed, only under this graphicality assumption we can solve the minimum problem in (1.10) in the class of surfaces which are graphs over the rectangles $\mathrm{R}_{i j}$. In turn, the graphicality of such area-minimizing surfaces allows to construct the sequence $\left\{u^{\varepsilon}\right\}$, see (4.11). Removing the graphicality assumption on $\Gamma$ requires some change of perspective and needs further investigation.

The content of the paper is the following. In Sect. 2.1, we recall some properties of functions of bounded variation of one variable and the definition of generalized graph (formula (2.1)). In Sect. 2.2, we recall some properties of Cartesian currents carried by a BV-function. The functional $\mathcal{G}$, appearing on the right-hand side of (1.10), is introduced in Definition 3.4. In Sect. 4 we show that

$$
\mathcal{A}(u, D) \leq|D|+\inf \left\{\mathcal{G}(\Gamma): \Gamma \in X_{\mathrm{Lip}}\right\},
$$

see Theorem 4.1. The proof is rather involved, mainly due to technical difficulties: we first start by supposing that the jump of $u$ consists of three segments (Proposition 4.4). Some work is required to define $u^{\varepsilon}$ on an $\varepsilon$-strip around the jump of $u$ and avoiding a neighborhood of the source triple junction (formula (4.11)) and to define $u^{\varepsilon}$ in the missing neighborhood of the source triple junction (step 3 of the proof of Proposition 4.4): the construction must be done in such a way that $u^{\varepsilon}$ remains Lipschitz, and turns out to be rather involved in the three triangles $T_{1}^{\varepsilon}, T_{2}^{\varepsilon}, T_{3}^{\varepsilon}$, see Fig. 4b. In Sect. 5 we prove that the infimum in (1.11) is a minimum. The proof is achieved by defining a topology in the space $X$ which allows to prove the density of $X_{\text {Lip }}$ in $X$ (Lemma 5.2), the continuity of the functional $\mathcal{G}$ (Proposition 5.4) and the sequential compactness of $X$ (Theorem 5.6). This latter result is also based on a uniform bound on the length of the connections (Proposition 5.3), which is a consequence of the graphicality assumptions on the connections.

\section{Some preliminaries}

In this section, we recall some results on functions of bounded variation of one variable [3], and on Cartesian currents [10], needed in the sequel.

\subsection{Functions of bounded variation in the interval}

Let $(a, b) \subset \mathbb{R}$ be a bounded open interval and $\varphi \in \mathrm{BV}((a, b))$; then

- $\varphi$ is bounded, and it is continuous up to an at most countable set of points of $(a, b)$ denoted by $J_{\varphi}$ (jump set);

- the right and left limits $\varphi\left(s_{ \pm}\right)$of $\varphi$ exist at any $s \in(a, b)$; the right limit $\varphi\left(a_{+}\right)$and the left limit $\varphi\left(b_{-}\right)$exist. Thus we may define

$$
\varphi_{+}(s):=\max \left\{\varphi\left(s_{+}\right), \varphi\left(s_{-}\right)\right\}, \quad \varphi_{-}(s):=\min \left\{\varphi\left(s_{+}\right), \varphi\left(s_{-}\right)\right\}, \quad s \in(a, b)
$$

- the distributional derivative $\varphi^{\prime}$ of $\varphi$ splits as

$$
\varphi^{\prime}=\dot{\varphi} \mathrm{d} s+\dot{\varphi}^{(j)}+\dot{\varphi}^{(c)},
$$

where $\dot{\varphi} \mathrm{d} s$ is the absolutely continuous part and $\dot{\varphi}$ is the approximate differential of $\varphi$ [3, p.138 and Cor. 3.33], $\dot{\varphi}^{(j)}$ and $\dot{\varphi}^{(c)}$ are the jump and the Cantor part, respectively. 
We shall always assume that $\varphi$ is a good representative in its $L^{1}$ class such that $\varphi(s)=\varphi_{+}(s)$ for all $s \in(a, b)$; the pointwise variation of $\varphi$ is equal to the total variation $\left|\varphi^{\prime}\right|((a, b))$.

The generalized graph of $\varphi$ is defined as

$$
\Gamma_{\varphi}:=\left\{\left(s, \theta \varphi\left(s_{-}\right)+(1-\theta) \varphi\left(s_{+}\right)\right): s \in(a, b), \theta \in[0,1]\right\},
$$

and the subgraph of $\varphi$ as

$$
S \mathcal{G}_{\varphi,(a, b)}:=\{(s, t) \in(a, b) \times \mathbb{R}: t \leq \varphi(s)\} .
$$

We recall that, if $\varphi \in L^{1}((a, b))$, then $\varphi \in \operatorname{BV}((a, b))$ if and only if $S \mathcal{G}_{\varphi,(a, b)}$ has finite perimeter in $(a, b) \times \mathbb{R}$. We denote by $\partial^{-} S \mathcal{G}_{\varphi,(a, b)}$ the reduced boundary of $S \mathcal{G}_{\varphi,(a, b)}$.

We conventionally set $\varphi\left(a_{-}\right)=0, \varphi\left(b_{+}\right)=0$; in this case we can define $\Gamma_{\varphi}$ as in (2.1) with $(a, b)$ replaced by $[a, b]$, hence the generalized graph will always pass through the end points of the interval (with possibly vertical parts over $a$ and $b$ ).

\subsection{Cartesian currents}

Let $I \subset \mathbb{R}$ be a bounded open interval and $\varphi \in \mathrm{BV}(I)$. We denote by

$$
\llbracket S \mathcal{G}_{\varphi, I} \rrbracket \in \mathcal{D}^{2}\left(\mathbb{R}^{2}\right)
$$

the 2-current in $\mathbb{R} \times \mathbb{R}$ defined as the integration over the subgraph $S \mathcal{G}_{\varphi, I}$. The current $\llbracket S \mathcal{G}_{\varphi, I} \rrbracket\llcorner I \times \mathbb{R}$ can be also identified with an integer multiplicity current in $I \times \mathbb{R}$; moreover $S \mathcal{G}_{\varphi, I}$ has finite perimeter in $I \times \mathbb{R}$ so, if $\partial \llbracket S \mathcal{G}_{\varphi, I} \rrbracket\llcorner I \times \mathbb{R}$ denotes the 1 -current in $I \times \mathbb{R}$ defined as the restriction to $I \times \mathbb{R}$ of the boundary of $\llbracket S \mathcal{G}_{\varphi, I} \rrbracket$, this results of finite mass.

For future purposes we recall the following result, see [10, Section 4.2.4].

Theorem 2.1 Let $\varphi \in \mathrm{BV}(I)$ and $\mathcal{T}$ be the current defined by

$$
\mathcal{T}:=-\partial \llbracket S \mathcal{G}_{\varphi, I} \rrbracket\llcorner I \times \mathbb{R} .
$$

Then $\mathcal{T} \in \mathcal{D}_{1}(I \times \mathbb{R})$ is a Cartesian current, and

$$
\mathcal{T}(\omega)=-\int<\omega(x), * \nu\left(x, S \mathcal{G}_{\varphi, I}\right)>d \mathcal{H}^{1}\left\llcorner\partial^{-} S \mathcal{G}_{\varphi, I}(x) \quad \forall \omega \in \mathcal{D}^{1}(I \times \mathbb{R}),\right.
$$

where $*$ is the Hodge operator and $v\left(\cdot, S \mathcal{G}_{\varphi, I}\right)$ is the inward generalized unit normal. Moreover $\mathcal{T}$ can be decomposed into three mutually singular currents

$$
\mathcal{T}=\mathcal{T}^{(a)}+\mathcal{T}^{(j)}+\mathcal{T}^{(c)},
$$

such that

$$
\begin{aligned}
& \mathcal{T}^{(a)}(\omega)=\int_{I}\left[\omega_{1}(s, \varphi(s))+\omega_{2}(s, \varphi(s)) \dot{\varphi}(s)\right] \mathrm{d} s, \\
& \mathcal{T}^{(j)}(\omega)=\sum_{s \in J_{\varphi}} n\left(s, J_{\varphi}\right) \int_{\varphi_{-}(s)}^{\varphi_{+}(s)} \omega_{2}(s, \sigma) \mathrm{d} \sigma, \\
& \mathcal{T}^{(c)}(\omega)=\int_{I} \omega_{2}(s, \varphi(s)) \dot{\varphi}^{(c)},
\end{aligned}
$$

where $\omega=\omega_{1} \mathrm{~d} s+\omega_{2} \mathrm{~d} \sigma$. 
The current $\mathcal{T}$ is boundaryless in $I \times \mathbb{R}$, namely $\partial \mathcal{T}=0$. Furthermore, if $\Gamma_{\varphi}$ is the generalized graph of $\varphi$ as defined in (2.1), it turns out that

$$
\partial^{-} S \mathcal{G}_{\varphi, I} \cap(I \times \mathbb{R}) \subseteq \Gamma_{\varphi}, \quad \mathcal{H}^{1}\left(\Gamma_{\varphi} \backslash \partial^{-} S \mathcal{G}_{\varphi, I}\right)=0 .
$$

It easily follows that the current $\mathcal{T}$ coincides with the integration over the rectifiable set $\Gamma_{\varphi}$ (with the correct orientation).

From now on, when the interval is clear from the context, we will simply denote $S \mathcal{G}_{\varphi, I}$ by $S \mathcal{G}_{\varphi}$.

\section{The functional $\mathcal{G}$}

In order to prove our main result (Theorem 1.1), we need some preparation. Take three open non-overlapping non-empty connected regions $E_{1}, E_{2}, E_{3}$ of an open disk $D$, each $E_{i}$ with non-empty interior and with $\overline{E_{1}} \cup \overline{E_{2}} \cup \overline{E_{3}}=\bar{D}$, and let $C_{i j}$ be their boundaries in $D$ as in the introduction.

Let $\alpha_{1}, \alpha_{2}, \alpha_{3}$ be the vertices of a closed triangle $\mathrm{T}$ as in Sect. 1; we suppose that $\mathrm{T}$ contains the origin $0_{\mathrm{T}}$ in its interior, and let $\ell_{i j}$ be as in (1.4).

Definition 3.1 (Connections in $\mathrm{T}$ ) We say that $\Gamma:=\left(\Gamma_{1}, \Gamma_{2}, \Gamma_{3}\right)$ is a $\mathrm{BV}$ graph-type (resp. Lip graph-type) connection in $\mathrm{T}$ if $\Gamma_{i}, i \in\{1,2,3\}$, are subsets of $\mathrm{T}$ such that $\Gamma_{1} \cap \Gamma_{2}=$ $\Gamma_{2} \cap \Gamma_{3}=\Gamma_{3} \cap \Gamma_{1}$ is one point $p$ of $\mathrm{T}$ called target triple point of $\Gamma, \alpha_{i} \in \Gamma_{i}$ for any $i=1,2,3$, and

$$
\Gamma_{i j}:=\Gamma_{i} \cup \Gamma_{j}, \quad i j \in\{12,23,31\},
$$

can be written as the generalized graph (resp. graph) of a function of bounded variation (resp. Lipschitz function) over the closed segment $\overline{\alpha_{i} \alpha_{j}}$ (see Figs. 1b, 13).

Note that the case $p \in \partial \mathrm{T}$ is not excluded. However, by definition, if $\pi_{i j}: \mathrm{T} \rightarrow \mathbb{R}_{\overline{\alpha_{i} \alpha_{j}}}, i j \in$ $\{12,23,31\}$, is the orthogonal projection on the line $\mathbb{R}_{\overline{\alpha_{i} \alpha_{j}}}$ containing $\overline{\alpha_{i} \alpha_{j}}$, then $\pi_{i j}(p) \in$ $\overline{\alpha_{i} \alpha_{j}}$. Set

$$
w_{i j}:=\left|\alpha_{i}-\pi_{i j}(p)\right| .
$$

If necessary, in the sequel we will often identify $\Gamma_{i j}$ with the (generalized) graph $\Gamma_{\varphi_{i j}}$ of a function

$$
\varphi_{i j}:\left[0, \ell_{i j}\right] \rightarrow[0, \operatorname{diamT}], \quad \varphi_{i j}=\varphi_{i j}\left(\Gamma_{i j}\right),
$$

of bounded variation. If $\mathrm{T}$ is acute, choosing a suitable cartesian coordinate system where the $s$-axis is the line $\mathbb{R}_{\overline{\alpha_{i} \alpha_{j}}}$, we necessarily have $\varphi_{i j}(0)=\varphi_{i j}\left(\ell_{i j}\right)=0$. In contrast, if the angle of $\mathrm{T}$ at $\alpha_{i}$ is greater than or equal to $\frac{\pi}{2}$ then $\varphi_{i j}$ might have a vertical part over $\alpha_{i}$ and $\varphi_{i j}\left(0_{+}\right)>0$.

In the sequel it will be often convenient to consider an extension of $\varphi_{i j}$ on $(-\infty, 0) \cup$ $\left(\ell_{i j},+\infty\right)$. This extension is denoted by $\tilde{\varphi}_{i j}$. In the case of acute triangle $\tilde{\varphi}_{i j}$ is always set equal to 0 on $(-\infty, 0) \cup\left(\ell_{i j},+\infty\right)$.

Remark 3.2 If for any $i j \in\{12,23,31\}, w_{i j}$ in (3.1) is a point of continuity of $\varphi_{i j}$ then the intersection of the generalized graph of $\varphi_{k i}$ with the set $\left[w_{k i}, \ell_{k i}\right] \times \mathbb{R}$ coincides with $\Gamma_{i}$ which is also the intersection of the generalized graph of $\varphi_{i j}$ with the set $\left[0, w_{i j}\right] \times \mathbb{R}$, where $i j, k i \in\{12,23,31\}, i j \neq k i$. If $w_{i j}$ is a jump point of $\varphi_{i j}$ this is in general not true, as in Fig. 14b, when $i=2$. 
Remark 3.3 Assume that an angle of $\mathrm{T}$ is greater than $\frac{\pi}{2}$, say for instance the angle at $\alpha_{1}$; as already said, the generalized graphs composing a connection $\Gamma$ are allowed to have vertical parts over $\alpha_{1}$. The target triple point $p$ of any connection $\Gamma$ belongs to $T_{\text {int }} \subset T$, the part of the triangle $\mathrm{T}$ which is enclosed between the two lines passing through $\alpha_{1}$ and orthogonal to $\overline{\alpha_{1} \alpha_{2}}$ and $\overline{\alpha_{1} \alpha_{3}}$, respectively.

Define the classes:

$$
\begin{aligned}
X_{\mathrm{Lip}} & :=\{\Gamma: \Gamma \mathrm{Lip} \text { graph }- \text { type connection in } \mathrm{T}\}, \\
X & :=\{\Gamma: \Gamma \mathrm{BV} \text { graph }- \text { type connection in } \mathrm{T}\} .
\end{aligned}
$$

Obviously $X_{\text {Lip }} \subset X$.

\subsection{Useful results on one-codimensional area-minimizing cartesian surfaces}

Let $\mathrm{R}_{i j}$ be as in (1.5), and $\Gamma \in X$. Then $\Gamma_{i j}, i j \in\{12,23,31\}$, are (generalized) graphs of functions $\varphi_{i j}$ of bounded variation over $\left[0, \ell_{i j}\right]$. Let $B \subset \mathbb{R}^{2}$ be an open disk containing the doubled rectangle $\widehat{\mathrm{R}}_{i j}$ defined as

$$
\widehat{\mathrm{R}}_{i j}:=\left[0, \ell_{i j}\right] \times\left[0,2 r_{i j}\right], \quad i j \in\{12,23,31\} .
$$

We use for simplicity the same notation $\varphi_{i j}$ for the extension of $\varphi_{i j}$ to $\widehat{\mathrm{R}}_{i j}$, defined as

$$
\varphi_{i j}(s, t)=\varphi_{i j}(s), \quad(s, t) \in \widehat{\mathrm{R}}_{i j} \quad i j \in\{12,23,31\},
$$

and for the extension of $\varphi_{i j}$ to a $W^{1,1}$ function on $B \backslash \widehat{\mathrm{R}}_{i j}$ as in [11, Theorem 2.16].

Let $\widehat{m}_{i j}=\widehat{m}_{i j}(\Gamma), i j \in\{12,23,31\}$, be a solution of the following Dirichlet minimum problem:

$$
\min \left\{\int_{\widehat{\mathrm{R}}_{i j}} \sqrt{1+|D f|^{2}}+\int_{\partial \widehat{\mathrm{R}}_{i j}}\left|f-\varphi_{i j}\right| d \mathcal{H}^{1}: f \in \mathrm{BV}(B), f=\varphi_{i j} \text { on } B \backslash \widehat{\mathrm{R}}_{i j}\right\},
$$

where $\int_{\widehat{R}_{i j}} \sqrt{1+|D f|^{2}}$ is the extension of the area functional to $\mathrm{BV}\left(\widehat{\mathrm{R}}_{i j}\right)$ as defined in [11, Definition 14.1 and Theorem 14.5].

From [11, Theorem 15.9] and the fact that the restriction of $\varphi_{i j}$ to $\partial \widehat{\mathrm{R}}_{i j}$ is continuous up to a countable set of points, it follows that $\widehat{m}_{i j}$ solves also

$$
\min \left\{\int_{\widehat{\mathrm{R}}_{i j}} \sqrt{1+|\nabla f|^{2}} \mathrm{~d} s \mathrm{~d} t: f \in W^{1,1}\left(\widehat{\mathrm{R}}_{i j}\right), f=\varphi_{i j} \mathcal{H}^{1}-\text { a.e. on } \partial \widehat{\mathrm{R}}_{i j}\right\},
$$

and it is unique by [11, Proposition 14.12]. Let $m_{i j}=m_{i j}(\Gamma)$ be the restriction of $\widehat{m}_{i j}$ to $\mathrm{R}_{i j}$. Then, by the symmetry of $\varphi_{i j}$ with respect to the line $\left\{t=r_{i j}\right\}, m_{i j}$ is the unique solution of the Dirichlet-Neumann minimum problem (1.7). From (1.6) it follows that the Dirichlet condition is zero on the sides $\{0\} \times\left[0, r_{i j}\right]$ and $\left\{\ell_{i j}\right\} \times\left[0, r_{i j}\right]$ of the rectangle $\mathrm{R}_{i j}$. Note that $m_{i j}$ is analytic in the interior of $\mathbf{R}_{i j}$ but not necessarily Lipschitz in $\mathbf{R}_{i j}$ [11, Theorem 14.13], see Fig. 2b.

Definition 3.4 (The functional $\mathcal{G}$ ) We define the functional $\mathcal{G}: X \longrightarrow[0,+\infty)$ as

$$
\mathcal{G}(\Gamma):=\mathfrak{A}_{12}(\Gamma)+\mathfrak{A}_{23}(\Gamma)+\mathfrak{A}_{31}(\Gamma),
$$

where $\mathfrak{A}_{i j}(\Gamma)$ are as in (1.8).

The properties of the functional $\mathcal{G}$ will be discussed in Sect. 5 . 


\section{Infimum of $\mathcal{G}$ as an upper bound of $\mathcal{A}(u, D)$}

The aim of this section is to provide the following upper bound for $\mathcal{A}(u, D)$.

Theorem 4.1 Let $u \in B V\left(D ;\left\{\alpha_{1}, \alpha_{2}, \alpha_{3}\right\}\right)$ be the function defined in (1.9). Then

$$
\mathcal{A}(u, D) \leq|D|+\inf \left\{\mathcal{G}(\Gamma): \Gamma \in X_{\text {Lip }}\right\} .
$$

It is not difficult to see, by truncating the area-minimizing surfaces graphs of $m_{i j}$ with the lateral boundary of the prisms $\left[0, \ell_{i j}\right] \times \mathrm{T}$, that the infimum in (4.1) is the same as the infimum obtained without requiring in Definition 3.1 that $\Gamma_{i} \subset \mathrm{T}, i \in\{1,2,3\}$.

Lemma 4.2 Let $\ell \geq 0, p \geq 0, \varphi \in \operatorname{Lip}([0, \ell] ;[0,+\infty))$ be such that $\varphi(0)=\varphi(\ell)=0$ and $w \in[0, \ell]$ so that $\varphi(w)=\mathrm{p}$. Then there exists a sequence $\left\{\varphi^{\sigma}\right\}$ of $\mathcal{C}^{\infty}$ equi-Lipschitz functions in $[0, \ell]$, converging to $\varphi$ uniformly on $[0, \ell]$ as $\sigma \rightarrow 0^{+}$, such that

$$
\varphi^{\sigma}(0)=\varphi^{\sigma}(\ell)=0, \quad \varphi^{\sigma}(w)=\mathrm{p}, \quad \text { for any } \sigma>0 \text { sufficiently small. }
$$

Proof Let us extend $\varphi$ in $\mathbb{R}$ such that $\varphi(s)=0$ in $\mathbb{R} \backslash[0, \ell]$, so that the extension (still denoted by $\varphi$ ) belongs to $\operatorname{Lip}(\mathbb{R})$. Let $\widehat{\varphi}^{\sigma}(s):=\eta_{\sigma} * \varphi$ in $\mathbb{R}$, where $\left\{\eta_{\sigma}\right\}$ is a standard sequence of mollifiers. Then $\widehat{\varphi}^{\sigma} \in \mathcal{C}^{\infty}(\mathbb{R}), \operatorname{Lip}\left(\widehat{\varphi}^{\sigma}\right) \leq \operatorname{Lip}(\varphi)$ and the sequence $\left\{\widehat{\varphi}^{\sigma}\right\}$ converges uniformly to $\varphi$ on compact subsets of $\mathbb{R}$. Without loss of generality, we may assume $\widehat{\varphi}^{\sigma}(s)=0$ in $\mathbb{R} \backslash(-\sigma / 2, \ell+\sigma / 2)$ and $\widehat{\varphi}^{\sigma}\left(\frac{\ell+2 \sigma}{\ell} w-\sigma\right)=\mathrm{p}+c_{\sigma}, c_{\sigma}=o(1)$. Let us first suppose $\mathrm{p} \neq 0$. We define

$$
\varphi^{\sigma}:[0, \ell] \rightarrow[0,+\infty), \quad \varphi^{\sigma}(s):=\frac{\mathrm{p}}{\mathrm{p}+c_{\sigma}} \widehat{\varphi}^{\sigma}\left(\frac{\ell+2 \sigma}{\ell} s-\sigma\right) .
$$

It is easy to see that $\varphi^{\sigma} \in \mathcal{C}^{\infty}([0, \ell]), \varphi^{\sigma}(0)=\varphi^{\sigma}(\ell)=0, \varphi^{\sigma}(w)=\mathrm{p}, \varphi^{\sigma}$ are equi-Lipschitz, and $\left\{\varphi^{\sigma}\right\}$ converges to $\varphi$ uniformly as $\sigma \rightarrow 0^{+}$. Notice that the obtained approximation is constantly null in a neighborhood of 0 and $\ell$.

In the case $\mathrm{p}=0$, we argue differently. We consider the two intervals $[0, w]$ and $[w, \ell]$ and we repeat the same approximation above in the single intervals; more precisely we choose two points $w_{1} \in(0, w)$ and $w_{2} \in(w, \ell)$ with $\varphi\left(w_{1}\right)>0, \varphi\left(w_{2}\right)>0$ (if these points do not exist it means that the functions are constantly 0 and they are already smooth, so there is nothing to prove). Then we approximate the two functions $\varphi\llcorner(0, w)$ and $\varphi\llcorner[w, \ell]$ as before, and we glue them along $w$. Note that the glued function is smooth in $w$ since both the two smooth approximations are constantly 0 in a neighborhood of $w$.

To prove Theorem 4.1 we use the three area-minimizing functions $m_{i j}$ introduced in Sect. 3.1, to construct a sequence $\left\{u^{\varepsilon}\right\}$ of Lipschitz functions that converges to $u$ in $L^{1}\left(D ; \mathbb{R}^{2}\right)$. However, $m_{i j}$ are only locally Lipschitz so we need the following smoothing lemma.

Lemma 4.3 Let $\Gamma \in X_{\text {Lip }}, i j \in\{12,23,31\}$. Let $\varphi_{i j}=\varphi_{i j}\left(\Gamma_{i j}\right) \in \operatorname{Lip}\left(\left[0, \ell_{i j}\right]\right), m_{i j}=$ $m_{i j}\left(\Gamma_{i j}\right) \in W^{1,1}\left(\mathrm{R}_{i j}\right)$, be defined as in Sect. 3.1. Then there exists a sequence $\left\{m_{i j}^{\sigma}\right\}$ of Lipschitz functions such that $m_{i j}^{\sigma}: \mathrm{R}_{i j} \rightarrow \mathbb{R}, m_{i j}^{\sigma}=\varphi_{i j}$ on $\partial_{D} \mathrm{R}_{i j}$, and

$$
\left|\int_{\mathrm{R}_{i j}} \sqrt{1+\left|\nabla m_{i j}\right|^{2}} \mathrm{~d} s \mathrm{~d} t-\int_{\mathrm{R}_{i j}} \sqrt{1+\left|\nabla m_{i j}^{\sigma}\right|^{2}} \mathrm{~d} s \mathrm{~d} t\right| \leq O(\sigma) .
$$

Proof This can be proved using an argument similar to the one in [4, p. 378; p. 381], and using also Lemma 4.2 with the choice $w=w_{i j}$ and $\mathrm{p}=\varphi_{i j}\left(w_{i j}\right)$. 
Fig. $3 E_{1}, E_{2}, E_{3}$ are separated by three segments of length $r_{12}$, $r_{23}, r_{31}$ that meet at the origin

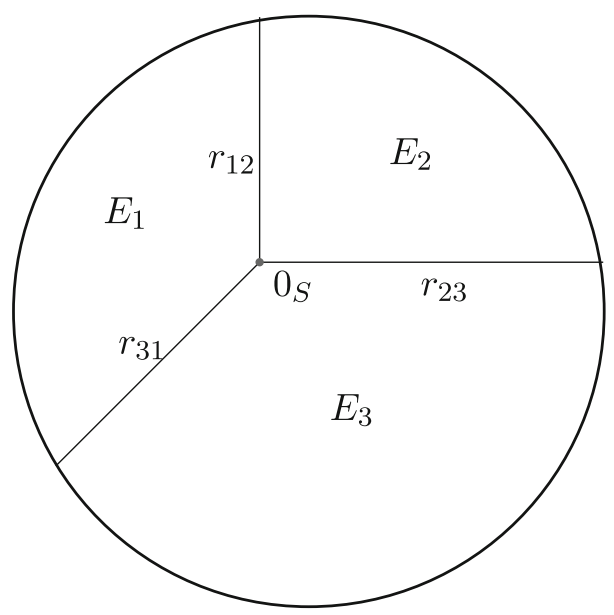

We start to prove Theorem 4.1 in the special case of a piecewise linear jump, as in Fig. 3.

Proposition 4.4 Let $u \in B V\left(D ;\left\{\alpha_{1}, \alpha_{2}, \alpha_{3}\right\}\right)$ be the map defined in (1.9) and assume that the jump set of $u$ consists of three distinct segments that meet at the origin. Then (4.1) holds.

Proof Let $\Gamma \in X_{\text {Lip }}$ be a connection passing through $p \in \mathrm{T}$ and $\mathcal{G}(\Gamma)=\mathfrak{A}_{12}(\Gamma)+\mathfrak{A}_{23}(\Gamma)+$ $\mathfrak{A}_{31}(\Gamma)$. To prove the proposition it is sufficient to construct a sequence $\left\{u^{\varepsilon}\right\} \subset \operatorname{Lip}\left(D ; \mathbb{R}^{2}\right)$ converging to $u$ in $L^{1}\left(D ; \mathbb{R}^{2}\right)$ such that

$$
\lim _{\varepsilon \rightarrow 0} \mathcal{A}\left(u^{\varepsilon}, D\right) \leq|D|+\mathfrak{A}_{12}(\Gamma)+\mathfrak{A}_{23}(\Gamma)+\mathfrak{A}_{31}(\Gamma) .
$$

Case 1 Assume that the segments separating $E_{1}, E_{2}, E_{3}$ meet at the origin with angles less than $\pi$, as in Fig. 3.

To simplify the computation we may assume that $p=0_{\mathrm{T}}$, see Fig. $1 \mathrm{~b}$. The idea of the proof is similar to the one used in [4], with however new difficulties, in particular in $T^{\varepsilon}$ (step $3)$. We will specify various subsets of $D$ and define the sequence $\left\{u^{\varepsilon}\right\}$ on each of these sets. Let $\varepsilon>0$ be sufficiently small and $\delta_{\varepsilon}>0$ be such that $\delta_{\varepsilon} \rightarrow 0^{+}$as $\varepsilon \rightarrow 0^{+}$. Define $\mathrm{T}^{\varepsilon}$ to be the triangle with the origin $0_{S}$ in its interior, with vertices $\zeta^{1}=\zeta_{\varepsilon}^{1}, \zeta^{2}=\zeta_{\varepsilon}^{2}$, and $\zeta^{3}=\zeta_{\varepsilon}^{3}$, and sides of lengths $\varepsilon_{12}, \varepsilon_{23}, \varepsilon_{31}, \varepsilon_{i j}:=\left|\zeta^{i}-\zeta^{j}\right|$; the sides of $\mathrm{T}^{\varepsilon}$ are perpendicular to the lines containing $r_{12}, r_{23}, r_{31}$ (respectively) and their distance from the origin $0_{S}$ equals $\delta_{\varepsilon}$. Define three cygar-shaped sets $S_{23}^{\varepsilon}, S_{31}^{\varepsilon}$ and $S_{12}^{\varepsilon}$ as in Fig. 4a: if for instance $y$ is a coordinate on $r_{12}$ and $x$ is the perpendicular coordinate, then $S_{12}^{\varepsilon}$ is defined as

$$
S_{12}^{\varepsilon}:=\left\{(x, y) \in D: x \in\left(\zeta_{1}^{1}, \zeta_{1}^{2}\right), y \geq \delta_{\varepsilon}\right\},
$$

where

$$
\zeta^{i}=\left(\zeta_{1}^{i}, \zeta_{2}^{i}\right), \quad i=1,2,3
$$

Let us set

$$
\begin{aligned}
E_{1}^{\varepsilon} & :=E_{1} \backslash\left(S_{31}^{\varepsilon} \cup T^{\varepsilon} \cup S_{12}^{\varepsilon}\right), \quad E_{2}^{\varepsilon}:=E_{2} \backslash\left(S_{23}^{\varepsilon} \cup T^{\varepsilon} \cup S_{12}^{\varepsilon}\right), \\
E_{3}^{\varepsilon} & :=E_{3} \backslash\left(S_{23}^{\varepsilon} \cup T^{\varepsilon} \cup S_{31}^{\varepsilon}\right) .
\end{aligned}
$$



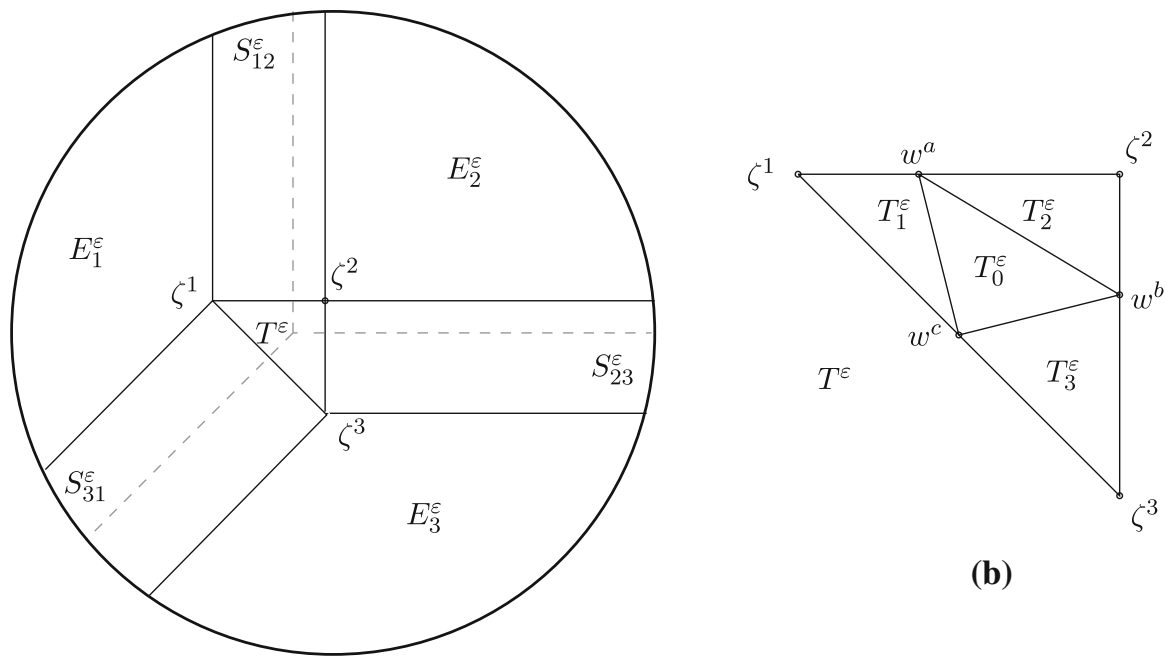

(a)

Fig. 4 a Case 1 of the proof of Proposition 4.4 and $\mathbf{b}$ zoom of $T^{\mathcal{E}}$ in (a)

Step 1 Definition of $u^{\varepsilon}$ on $E_{1}^{\varepsilon} \cup E_{2}^{\varepsilon} \cup E_{3}^{\varepsilon}$. We define

$$
u^{\varepsilon}:= \begin{cases}\alpha_{1} & \text { in } E_{1}^{\varepsilon}, \\ \alpha_{2} & \text { in } E_{2}^{\varepsilon}, \\ \alpha_{3} & \text { in } E_{3}^{\varepsilon} .\end{cases}
$$

Note that $\mathcal{A}\left(u^{\varepsilon}, E_{1}^{\varepsilon} \cup E_{2}^{\varepsilon} \cup E_{3}^{\varepsilon}\right)=\left|E_{1}^{\varepsilon}\right|+\left|E_{2}^{\varepsilon}\right|+\left|E_{3}^{\varepsilon}\right|$, hence

$$
\lim _{\varepsilon \rightarrow 0^{+}} \mathcal{A}\left(u^{\varepsilon}, E_{1}^{\varepsilon} \cup E_{2}^{\varepsilon} \cup E_{3}^{\varepsilon}\right)=|D| .
$$

Step 2 Definition of $u^{\varepsilon}$ on $S_{23}^{\varepsilon} \cup S_{31}^{\varepsilon} \cup S_{12}^{\varepsilon}$.

We will start with the construction on $S_{12}^{\varepsilon}$. Set

$$
\xi=\left(\xi_{1}, \xi_{2}\right):=\frac{\alpha_{2}-\alpha_{1}}{\ell_{12}} \in \mathbb{S}^{1}, \quad \eta=\left(\eta_{1}, \eta_{2}\right):=\xi^{\perp},
$$

where ${ }^{\perp}$ denotes the counterclockwise rotation of $\pi / 2$.

Let $\psi_{12}^{\varepsilon}:\left[\delta_{\varepsilon}, r_{12}+c_{\varepsilon}\right] \rightarrow\left[0, r_{12}\right]$ be linear, increasing, surjective, where $c_{\varepsilon}>0$ is the smallest number such that

$$
S_{12}^{\varepsilon} \subset\left[\zeta_{1}^{1}, \zeta_{1}^{2}\right] \times\left[\delta_{\varepsilon}, r_{12}+c_{\varepsilon}\right], \lim _{\varepsilon \rightarrow 0^{+}} c_{\varepsilon}=0 .
$$

Note that for any $y \in\left[\delta_{\varepsilon}, r_{12}+c_{\varepsilon}\right]$ we have

$$
\left(\psi_{12}^{\varepsilon}\right)^{\prime}(y)=\frac{r_{12}}{r_{12}+c_{\varepsilon}-\delta_{\varepsilon}}=: \kappa_{\varepsilon}, \quad \lim _{\varepsilon \rightarrow 0^{+}} \kappa_{\varepsilon}=1 .
$$

Let $m_{12}^{\sigma}$ be the map defined in Lemma 4.3, whose area on $\mathrm{R}_{12}$ is by construction close to $\mathfrak{A}_{12}(\Gamma)$, with $\left\{\sigma_{\varepsilon}\right\} \subset(0,+\infty)$ a sequence such that

$$
\lim _{\varepsilon \rightarrow 0^{+}} \sigma_{\varepsilon}=0
$$


We set, with $\sigma=\sigma_{\varepsilon}$ for simplicity,

$$
u^{\varepsilon}(x, y):=\alpha_{1}+\left(\frac{x-\zeta_{1}^{1}}{\varepsilon_{12}}\right) \ell_{12} \xi+m_{12}^{\sigma}\left(\frac{x-\zeta_{1}^{1}}{\varepsilon_{12}} \ell_{12}, \psi_{12}^{\varepsilon}(y)\right) \eta, \quad(x, y) \in S_{12}^{\varepsilon} .
$$

Observe that $u^{\varepsilon}=\left(u_{1}^{\varepsilon}, u_{2}^{\varepsilon}\right) \in \operatorname{Lip}\left(S_{12}^{\varepsilon} ; \mathbb{R}^{2}\right), u^{\varepsilon}=\alpha_{1}$ on $\left\{(x, y) \in S_{12}^{\varepsilon}: x=\zeta_{1}^{1}\right\}$, and $u^{\varepsilon}=\alpha_{2}$ on $\left\{(x, y) \in S_{12}^{\varepsilon}: x=\zeta_{1}^{2}\right\}$. By the definition of $m_{12}^{\sigma}$, it is uniquely defined the point (depending on $\varepsilon$ ) $w^{a}=\left(w_{1}^{a}, w_{2}^{a}\right) \in \overline{\zeta^{1} \zeta^{2}}$ such that $u^{\varepsilon}\left(w_{1}^{a}, w_{2}^{a}\right)=0_{T}$ (see Fig. 4b). Write for simplicity

$$
\tilde{m}=m_{12}^{\sigma} \text {. }
$$

Using that $|\xi|=|\eta|=1, \xi_{1} \eta_{1}+\xi_{2} \eta_{2}=0$, and $\xi_{1} \eta_{2}-\xi_{2} \eta_{1}=1$, we compute

$$
\begin{aligned}
1 & +\left|\nabla u_{1}^{\varepsilon}\right|^{2}+\left|\nabla u_{2}^{\varepsilon}\right|^{2}+\left(\frac{\partial u_{1}^{\varepsilon}}{\partial x} \frac{\partial u_{2}^{\varepsilon}}{\partial y}-\frac{\partial u_{1}^{\varepsilon}}{\partial y} \frac{\partial u_{2}^{\varepsilon}}{\partial x}\right)^{2} \\
& =1+\frac{\ell_{12}^{2}}{\varepsilon_{12}^{2}}\left(1+\left(\tilde{m}_{S}\right)^{2}+\left(\tilde{m}_{t}\right)^{2} \kappa_{\varepsilon}^{2}\left(1+\frac{\varepsilon_{12}^{2}}{\ell_{12}^{2}}\right)\right),
\end{aligned}
$$

where $\tilde{m}_{s}, \tilde{m}_{t}$ denote, respectively, the partial derivatives of $\tilde{m}$ with respect to $s:=\frac{x-\zeta_{1}^{1}}{\varepsilon_{12}} \ell_{12}$ and $t:=\psi_{12}^{\varepsilon}(y)$, and are evaluated at $\left(\frac{x-\zeta_{1}^{1}}{\varepsilon_{12}} \ell_{12}, \psi_{12}^{\varepsilon}(y)\right)$. As a consequence

$$
\begin{aligned}
& \mathcal{A}\left(u^{\varepsilon}, S_{12}^{\varepsilon}\right) \\
& =\frac{\ell_{12}}{\varepsilon_{12}} \int_{S_{12}^{\varepsilon}} \sqrt{1+\left[\widetilde{m}_{s}\left(\frac{x-\zeta_{1}^{1}}{\varepsilon_{12}} \ell_{12}, \psi_{12}^{\varepsilon}(y)\right)\right]^{2}+\left[\widetilde{m}_{t}\left(\frac{x-\zeta_{1}^{1}}{\varepsilon_{12}} \ell_{12}, \psi_{12}^{\varepsilon}(y)\right)\right]^{2} \kappa_{\varepsilon}^{2}\left(1+\frac{\varepsilon_{12}^{2}}{\ell_{12}^{2}}\right)+O\left(\varepsilon^{2}\right) \mathrm{d} x \mathrm{~d} y} \\
& =\frac{1}{\kappa_{\varepsilon}} \int_{\mathrm{R}_{12} \backslash P_{\varepsilon}} \sqrt{1+\left[\widetilde{m}_{s}(s, t)\right]^{2}+\left[\widetilde{m}_{t}(s, t)\right]^{2} \kappa_{\varepsilon}^{2}\left(1+\frac{\varepsilon_{12}^{2}}{\ell_{12}^{2}}\right)+O\left(\varepsilon^{2}\right) \mathrm{d} s \mathrm{~d} t,}
\end{aligned}
$$

where the last equality follows by the change of variables

$$
\begin{aligned}
\Phi: \mathrm{R}_{12} \ni(s, t) \mapsto \Phi(s, t): & =\left(\frac{\varepsilon_{12}}{\ell_{12}} s+\zeta_{1}^{1}, \psi_{12}^{\varepsilon}{ }^{-1}(t)\right) \\
& =(x, y) \in\left[\zeta_{1}^{1}, \zeta_{1}^{2}\right] \times\left[\delta_{\varepsilon}, r_{12}+c_{\varepsilon}\right] \supset S_{12}^{\varepsilon},
\end{aligned}
$$

and $P_{\varepsilon}:=\mathrm{R}_{12} \backslash \Phi^{-1}\left(S_{12}^{\varepsilon}\right)$ (see Fig. 5). Hence, recalling also (4.9), we conclude

$$
\lim _{\varepsilon \rightarrow 0^{+}} \mathcal{A}\left(u^{\varepsilon}, S_{12}^{\varepsilon}\right)=\int_{\mathrm{R}_{12}} \sqrt{1+\left(\tilde{m}_{s}\right)^{2}+\left(\tilde{m}_{t}\right)^{2}} \mathrm{~d} s \mathrm{~d} t .
$$

We recall that from (4.3) it follows that

$$
\int_{\mathrm{R}_{12}} \sqrt{1+\left(\tilde{m}_{s}\right)^{2}+\left(\tilde{m}_{t}\right)^{2}} \mathrm{~d} s \mathrm{~d} t=\mathfrak{A}_{12}(\Gamma)+O(\varepsilon) .
$$

Hence, employing the same construction in the strips $S_{23}^{\varepsilon}$ and $S_{31}^{\varepsilon}$ we obtain

$$
\lim _{\varepsilon \rightarrow 0^{+}} \mathcal{A}\left(u^{\varepsilon}, S_{23}^{\varepsilon} \cup S_{31}^{\varepsilon} \cup S_{12}^{\varepsilon}\right)=\mathfrak{A}_{12}(\Gamma)+\mathfrak{A}_{23}(\Gamma)+\mathfrak{A}_{31}(\Gamma) .
$$


Fig. 5 The set $P_{\varepsilon}$ is bounded by the bold contour

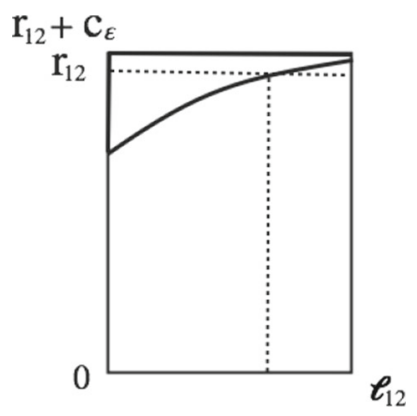

Step 3 Definition of $u^{\varepsilon}$ on $T^{\varepsilon}$. We divide $T^{\varepsilon}$ into four closed triangles $T_{1}^{\varepsilon}, T_{2}^{\varepsilon}, T_{3}^{\varepsilon}$ and $T_{0}^{\varepsilon}$ as in Fig. 4b. We set

$$
u^{\varepsilon}:=0_{\mathrm{T}} \text { in } T_{0}^{\varepsilon} .
$$

We first define $u^{\varepsilon}$ on $\partial T_{1}^{\varepsilon}$ as follows:

(i) the value of $u^{\varepsilon}$ at $\zeta^{1}$ is $\alpha_{1}$;

(ii) the value of $u^{\varepsilon}$ on the side $\overline{w^{c} w^{a}}$ is $0_{\mathrm{T}}$.

Note that $u^{\varepsilon}$ is already defined on the edges $\overline{\zeta^{1} w^{a}}$ and $\overline{\zeta^{1} w^{c}}$ and its graph over both edges is given by a rescaled version of the curve $\Gamma_{1}$ suitably parametrized.

More precisely, we recall that $\pi_{12}: \Gamma_{1} \rightarrow \overline{\alpha_{1} \alpha_{2}}$ and $\pi_{31}: \Gamma_{1} \rightarrow \overline{\alpha_{3} \alpha_{1}}$ are the orthogonal projections onto the edges $\overline{\alpha_{1} \alpha_{2}}$ and $\overline{\alpha_{3} \alpha_{1}}$. Since $\Gamma_{1}$ is, by hypothesis, a part of a Lipschitz graph, the maps $\pi_{12}\left\llcorner\Gamma_{1}\right.$ and $\pi_{31}\left\llcorner\Gamma_{1}\right.$ are bi-Lipschitz bijections between $\Gamma_{1}$ and the segments $\overline{\alpha_{1} \pi_{12}(p)}$ and $\overline{\alpha_{1} \pi_{31}(p)}$, respectively. We know that if $(s, t)$ are coordinates on $\mathrm{T}$ with respect to the system with $s$-axis $\overline{\alpha_{1} \alpha_{2}}$, then the inverse of $\pi_{12} L \Gamma_{1}$ is given by $\Phi_{12}: \overline{\alpha_{1} \alpha_{2}} \rightarrow \Gamma_{1}$,

$$
\Phi_{12}((s, 0))=\left(s, \varphi_{12}(s)\right) .
$$

Let us denote by $L_{12}=L_{12}^{\varepsilon}: \overline{\zeta^{1} w^{a}} \subset \mathbb{R}_{S}^{2} \rightarrow \overline{\alpha_{1} \pi_{12}(p)} \subset \mathbb{R}_{T}^{2}$ and $L_{31}=L_{31}^{\varepsilon}: \overline{\zeta^{1} w^{a}} \subset$ $\mathbb{R}_{S}^{2} \rightarrow \overline{\alpha_{1} \pi_{31}(p)} \subset \mathbb{R}_{T}^{2}$ the linear bijective maps

$$
L_{12}(Q)=\alpha_{1}+\frac{\left|Q-\zeta^{1}\right|}{\varepsilon_{12}}\left(\alpha_{2}-\alpha_{1}\right), \quad L_{31}\left(Q^{\prime}\right)=\alpha_{1}+\frac{\left|Q^{\prime}-\zeta^{1}\right|}{\varepsilon_{31}}\left(\alpha_{3}-\alpha_{1}\right) .
$$

Then we define

$$
u^{\varepsilon}:=\Phi_{12} \circ L_{12} \quad \text { on } \overline{\zeta^{1} w^{a}},
$$

and

$$
u^{\varepsilon}=\Phi_{31} \circ L_{31} \quad \text { on } \overline{\zeta^{1} w^{c}} .
$$

Compare formula (4.11). Since $\Phi_{12}$ and $\Phi_{31}$ are Lipschitz with Lipschitz constants independent of $\varepsilon$, and the Lipschitz constants of $L_{12}$ and $L_{31}$ have order $\frac{1}{\varepsilon}$, it follows that the Lipschitz constants of $u^{\varepsilon}$ over the segments $\overline{\zeta^{1} w^{a}}$ and $\overline{\zeta^{1} w^{c}}$ have order $\frac{1}{\varepsilon}$.

Now we want to define $u^{\varepsilon}$ in the interior of $T_{1}^{\varepsilon}$. First we observe that the map $\pi_{31} \circ \Phi_{12}$ : $\overline{\alpha_{1} \pi_{12}(p)} \subset \mathbb{R}_{T}^{2} \rightarrow \overline{\alpha_{1} \pi_{31}(p)} \subset \mathbb{R}_{T}^{2}$ is a bi-Lipschitz bijection, with constant independent 
of $\varepsilon$. A direct computation then provides that the map $\Psi: \overline{\zeta^{1} w^{a}} \subset \mathbb{R}_{S}^{2} \rightarrow \overline{\zeta^{1} w^{c}} \subset \mathbb{R}_{S}^{2}$ defined by

$$
\Psi:=\left(L_{31}\right)^{-1} \circ \pi_{31} \circ \Phi_{12} \circ L_{12},
$$

is bi-Lipschitz between $\overline{\zeta^{1} w^{a}}$ and $\overline{\zeta^{1} w^{c}}$ with bi-Lipschitz constant of order 1 as $\varepsilon \rightarrow 0^{+}$. Given $Q \in \overline{\zeta^{1} w^{a}}$ let $Q^{\prime}:=\Psi(Q) \in \overline{\zeta^{1} w^{c}}$.

Now we show that $T_{1}^{\varepsilon}$ is foliated by the segments $\overline{Q Q^{\prime}}$, i.e., for any $R \in T_{1}^{\varepsilon}$ we can find a unique $Q \in \overline{\zeta^{1} w^{a}}$ for which $R \in \overline{Q Q^{\prime}}$.

First we notice that $\overline{Q Q^{\prime}} \cap \overline{S S^{\prime}}=\emptyset$ for any $Q \neq S \in \overline{\zeta^{1} w^{a}}$ with $Q^{\prime}=\Psi(Q)$ and $S^{\prime}=\Psi(S)$. Indeed, thanks to the fact that $\Psi$ is a homeomorphism and that it keeps $\zeta^{1}$ fixed, it is easy to see that if $S \in \overline{\zeta^{1} Q}$ then $S^{\prime} \in \overline{\zeta^{1} Q^{\prime}}$, or if $Q \in \overline{\zeta^{1} S}$ then $Q^{\prime} \in \overline{\zeta^{1} S^{\prime}}$. Consider the function

$$
f(q, \sigma)=q \tau+\sigma v(q), \quad q \in\left[0,\left|w^{a}-\zeta^{1}\right|\right], \sigma \in[0,|\Psi(q \tau)-q \tau|],
$$

where $\tau:=\frac{w^{a}-\zeta^{1}}{\left|w^{a}-\zeta^{1}\right|}$ and $\nu(q):=\frac{\Psi(q \tau)-q \tau}{|\Psi(q \tau)-q \tau|}$. It is clear that the image of $f$ is a closed set and $\operatorname{Im}(f)=\left\{\overline{Q Q^{\prime}}: Q \in \overline{\zeta^{1} w^{a}}, Q^{\prime}=\Psi(Q)\right\}$. Now we show that $\operatorname{Im}(f)=T_{1}^{\varepsilon}$. Assume by contradiction there is $R \in T_{1}^{\varepsilon} \backslash \operatorname{Im}(f)$ and take a disk $B \subset T_{1}^{\varepsilon} \backslash \operatorname{Im}(f)$ centered at $R$. Let $Q_{r}, Q_{l} \in \overline{\zeta^{1} w^{a}}$ be such that $q_{r}:=\left|Q_{r}-\zeta^{1}\right|$ (resp. $q_{l}=\left|Q_{l}-\zeta^{1}\right|$ ) be the supremum (resp. the infimum) parameter for which $B$ lies on the right (resp. left) of $\overline{Q_{r} Q_{r}^{\prime}}$ (resp. $\overline{Q_{l} Q_{l}^{\prime}}$ ). Note that $Q_{r} \neq Q_{l}$ due to the injectivity of $\Psi$, thus for any $Q \in \overline{Q_{r} Q_{l}}$ the segment $\overline{Q Q^{\prime}}$ must intersect $B$, a contradiction, see Fig. 6 a.

Hence we may define $u^{\varepsilon}$ on $T_{1}^{\varepsilon}$ as

$$
u^{\varepsilon}(R):=u^{\varepsilon}(Q), \quad R \in \overline{Q Q^{\prime}}, Q \in \overline{\zeta^{1} w^{a}} .
$$

We want now to show that on $T_{1}^{\varepsilon}, u^{\varepsilon}$ is Lipschitz continuous with Lipschitz constant of order $\frac{1}{\varepsilon}$. To prove this let us fix $R \in T_{1}^{\varepsilon}$. By definition $u^{\varepsilon}(R)=u^{\varepsilon}(Q)$ for some $Q \in \overline{\zeta^{1} w^{a}}$ and $u^{\varepsilon}$ is constant on the segment $\overline{Q Q^{\prime}} \ni R$.

Let $e: T_{1}^{\varepsilon} \rightarrow \overline{\zeta^{1} w^{a}}$ be the function taking $(x, y) \in T_{1}^{\varepsilon}$ to the intersection point of $\overline{\zeta^{1} w^{a}}$ and the line passing through $(x, y)$ parallel to $\overline{Q Q^{\prime}}$. Let $g: T_{1}^{\varepsilon} \rightarrow \overline{\zeta^{1} w^{c}}$ be the function taking $(x, y) \in T_{1}^{\varepsilon}$ to the intersection point of $\overline{\zeta^{1} w^{c}}$ and the line passing through $(x, y)$ parallel to $\overline{Q Q^{\prime}}$. Let $\hat{R} \in T_{1}^{\varepsilon}$ be a point in $T_{1}^{\varepsilon}$; we want to estimate the ratio

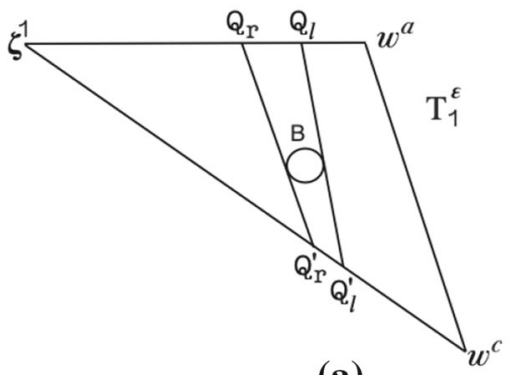

(a)

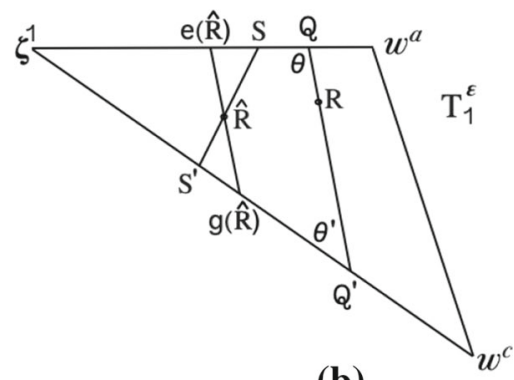

(b)

Fig. 6 Surjectivity of the foliation in $T_{1}^{\varepsilon} \subset \mathbb{R}_{S}^{2}$ 


$$
\frac{\left|u^{\varepsilon}(\hat{R})-u^{\varepsilon}(R)\right|}{|\hat{R}-R|} .
$$

Consider the two segments $\overline{Q e(\hat{R})}$ and $\overline{Q^{\prime} g(\hat{R})}$. By definition $\hat{R} \in \overline{S S^{\prime}}$ and $u^{\varepsilon}(\hat{R})=u^{\varepsilon}(S)=$ $u^{\varepsilon}\left(S^{\prime}\right)$ for two points $S \in \overline{\zeta^{1} w^{a}}$ and $S^{\prime} \in \overline{\zeta^{1} w^{c}}$. It is straightforward that either $S \in \overline{Q e(\hat{R})}$ or $S^{\prime} \in \overline{Q^{\prime} g(\hat{R})}$. Without loss of generality suppose the first case holds, see Fig. 6 b.

Finally, denote by $\theta$ the angle between $\overline{Q Q^{\prime}}$ and $\overline{\zeta^{1} w^{a}}$ and by $\theta^{\prime}$ the angle between $\overline{Q Q^{\prime}}$ and $\overline{\zeta^{1} w^{c}}$. Using the fact that the homeomorphism in (4.19) is bi-Lipschitz with constant of order 1 it is not difficult to see that there is a constant $\theta_{0}>0$ independent of $\varepsilon$ such that $\min \left\{\theta, \theta^{\prime}\right\} \geq \theta_{0}$. This is a consequence of the fact that the bi-Lipschitz constant of $\Psi$ in (4.19) is of order 1 . Indeed, if $L=\operatorname{lip}(\Psi)$ and $1 / L^{\prime}=\operatorname{lip}\left(\Psi^{-1}\right)$, we see that

$$
L^{\prime} \leq \frac{\left|Q^{\prime}-\zeta^{1}\right|}{\left|Q-\zeta^{1}\right|} \leq L
$$

hence

$$
\frac{1 / L+\cos \theta_{\zeta^{1}}}{\sin \theta_{\zeta^{1}}} \leq \frac{\cos \theta}{\sin \theta} \leq \frac{1 / L^{\prime}+\cos \theta_{\zeta^{1}}}{\sin \theta_{\zeta^{1}}},
$$

where $\theta_{\zeta^{1}}$ is the angle at $\zeta^{1}$ (here we have used the law of sines and that $\theta^{\prime}=\pi-\theta_{\zeta^{1}}-\theta$ ). A similar estimate holding for $\theta^{\prime}$, this readily provides the boundedness from below of $\min \left\{\theta, \theta^{\prime}\right\}$.

As a consequence we have

$$
|\hat{R}-R| \geq|Q-e(\hat{R})||\sin \theta| \geq|Q-e(\hat{R})|\left|\sin \theta_{0}\right| .
$$

Thus, we compute

$$
\frac{\left|u^{\varepsilon}(\hat{R})-u^{\varepsilon}(R)\right|}{|\hat{R}-R|} \leq \frac{\left|u^{\varepsilon}(Q)-u^{\varepsilon}(S)\right|}{|Q-e(\hat{R})||\sin \theta|} \leq \frac{\left|u^{\varepsilon}(Q)-u^{\varepsilon}(S)\right|}{|Q-S||\sin \theta|} \leq \frac{1}{\left|\sin \theta_{0}\right|} \frac{\left|u^{\varepsilon}(Q)-u^{\varepsilon}(S)\right|}{|Q-S|},
$$

that is bounded by the Lipschitz constant of $\Phi_{12} \circ L_{12}$ which is of order $\frac{1}{\varepsilon}$.

Eventually we compute the Jacobian of $u^{\varepsilon}$ in (4.20). By construction the image of $T_{1}^{\varepsilon}$ by $u^{\varepsilon}$ is exactly the curve $\Gamma_{1}$, which has zero Lebesgue measure in $\mathbb{R}^{2}$. By a standard application of the area formula it follows that the Jacobian of $u^{\varepsilon}$ is vanishes a.e. in $T_{1}^{\varepsilon}$. We have concluded the definition of $u^{\varepsilon}$ in $T_{1}^{\varepsilon}$. The constructions on $T_{2}^{\varepsilon}$ and on $T_{3}^{\varepsilon}$ are similar, and similar estimates of the derivatives and Jacobians hold.

Using that the area of the triangle $T^{\varepsilon}$ is of order $\varepsilon^{2}$, we have

$$
\lim _{\varepsilon \rightarrow 0^{+}} \mathcal{A}\left(u^{\varepsilon}, T_{\varepsilon}\right)=\lim _{\varepsilon \rightarrow 0^{+}}\left(O(\varepsilon)+O\left(\varepsilon^{2}\right)\right)=0 .
$$

From (4.7), (4.11), (4.16), (4.20), and the estimates above it follows that

$$
\left\{u^{\varepsilon}\right\} \subset \operatorname{Lip}\left(D ; \mathbb{R}^{2}\right), \quad \lim _{\varepsilon \rightarrow 0^{+}} \int_{D}\left|u^{\varepsilon}-u\right| \mathrm{d} x \mathrm{~d} y=0 .
$$

Moreover

$\mathcal{A}\left(u^{\varepsilon}, D\right)=\mathcal{A}\left(u^{\varepsilon}, E_{1}^{\varepsilon} \cup E_{2}^{\varepsilon} \cup E_{3}^{\varepsilon}\right)+\mathcal{A}\left(u^{\varepsilon}, S_{23}^{\varepsilon}\right)+\mathcal{A}\left(u^{\varepsilon}, S_{31}^{\varepsilon}\right)+\mathcal{A}\left(u^{\varepsilon}, S_{12}^{\varepsilon}\right)+\mathcal{A}\left(u^{\varepsilon}, T^{\varepsilon}\right)$. 


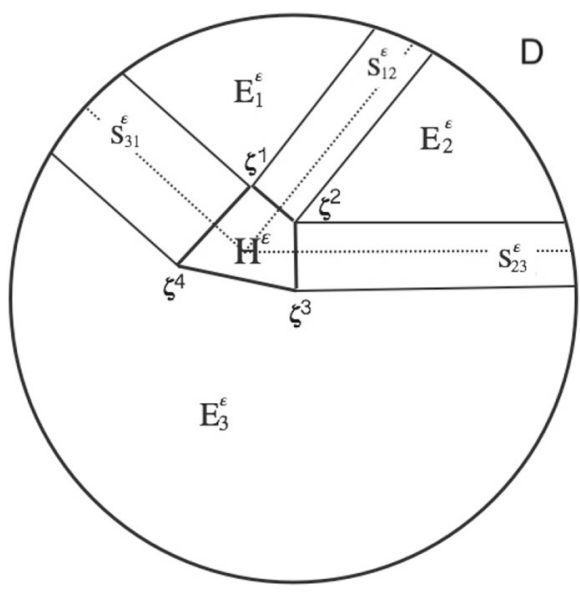

(a)

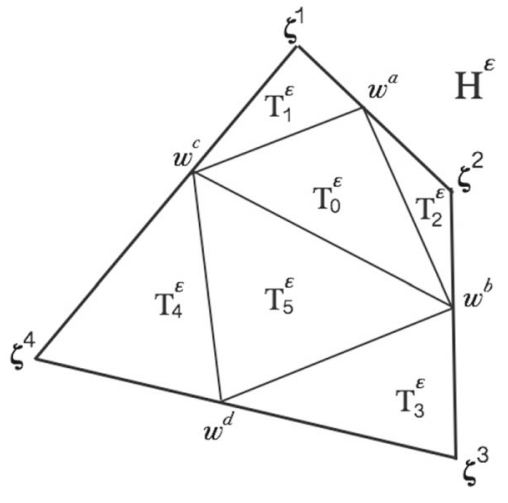

(b)

Fig. 7 a The non-acute case and $\mathbf{b}$ zoom of $H^{\varepsilon}$ in (a)

Then (4.4) follows from (4.24), (4.8), (4.15), (4.10) and (4.22).

Case 2 Assume that two of the segments separating $E_{1}, E_{2}, E_{3}$ meet at the origin with an angle greater than or equal to $\pi$.

Similar to Case 1, we divide the domain $D$ into a finite number of subsets and define the sequence $\left\{u^{\varepsilon}\right\}$ on each of these sets. Draw the normal to each segment at the point of distance $\delta_{\varepsilon}$ from the origin. The normal lines meet at two points $\zeta^{1}, \zeta^{2}$. Divide $D$ into three cygar-shape subsets $S_{23}^{\varepsilon}, S_{31}^{\varepsilon}, S_{12}^{\varepsilon}$ (with widths of order $\delta_{\varepsilon}=O(\varepsilon)$ ) and a quadrilateral $H^{\varepsilon}$ as in Fig. 7 a.

Let

$$
\begin{aligned}
E_{1}^{\varepsilon} & :=E_{1} \backslash\left(S_{31}^{\varepsilon} \cup H^{\varepsilon} \cup S_{12}^{\varepsilon}\right), \quad E_{2}^{\varepsilon}:=E_{2} \backslash\left(S_{23}^{\varepsilon} \cup H^{\varepsilon} \cup S_{12}^{\varepsilon}\right), \\
E_{3}^{\varepsilon} & :=E_{3} \backslash\left(S_{23}^{\varepsilon} \cup H^{\varepsilon} \cup S_{31}^{\varepsilon}\right) .
\end{aligned}
$$

Set

$$
u^{\varepsilon}:= \begin{cases}\alpha_{1} & \text { in } E_{1}^{\varepsilon}, \\ \alpha_{2} & \text { in } E_{2}^{\varepsilon}, \\ \alpha_{3} & \text { in } E_{3}^{\varepsilon} .\end{cases}
$$

Define $u^{\varepsilon}$ on $S_{23}^{\varepsilon} \cup S_{31}^{\varepsilon} \cup S_{12}^{\varepsilon}$ as in Step 2 in case 1 . It remains to define $u^{\varepsilon}$ on $H^{\varepsilon}$. Recall that by construction there exist uniquely determined three points $w^{a} \in \overline{\zeta^{1} \zeta^{2}}, w^{b} \in \overline{\zeta^{2} \zeta^{3}}$ and $w^{c} \in \overline{\zeta^{1} \zeta^{4}}$ such that

$$
u^{\varepsilon}\left(w^{a}\right)=u^{\varepsilon}\left(w^{b}\right)=u^{\varepsilon}\left(w^{c}\right)=0_{\mathrm{T}} .
$$

Divide $H^{\varepsilon}$ into six triangles $T_{0}^{\varepsilon}, T_{1}^{\varepsilon}, T_{2}^{\varepsilon}, T_{3}^{\varepsilon}, T_{4}^{\varepsilon}, T_{5}^{\varepsilon}$, as in Fig. 7b, where $w^{d}$ is any point in $\overline{\zeta^{3} \zeta^{4}}$ and $w^{d} \neq \zeta^{3}, w^{d} \neq \zeta^{4}$.

Set

$$
u^{\varepsilon}:=0_{\mathrm{T}} \text { in } T_{0}^{\varepsilon} .
$$


We define $u^{\varepsilon}$ in the triangles $T_{1}^{\varepsilon}$ and $T_{2}^{\varepsilon}$ as in Step 3; it remains to define $u^{\varepsilon}$ on $T_{3}^{\varepsilon}, T_{4}^{\varepsilon}, T_{5}^{\varepsilon}$. Let us first define $u^{\varepsilon}$ on the edges $\overline{w^{c} w^{d}}$ and $\overline{w^{b} w^{d}}$. The map $u^{\varepsilon}$ is already defined on the other edges, and its graph over $\overline{\zeta^{4} w^{c}}$ and $\overline{\zeta^{3} w^{b}}$ is given by a suitable reparametrization of the curve $\Gamma_{3}$, whereas $u^{\varepsilon}$ on $\overline{\zeta^{4} \zeta^{3}}$ is constantly $\alpha_{3}$. Therefore it suffices to define $u^{\varepsilon}$ in such a way that its graph over $\overline{w^{c} w^{d}}$ and $\overline{w^{b} w^{d}}$ coincides with $\Gamma_{3}$ as well, and then we can define $u^{\varepsilon}$ inside $T_{3}^{\varepsilon}$ and $T_{4}^{\varepsilon}$ using the same construction for $T_{1}^{\varepsilon}$ in step 3. Similarly, using that the graph of $u^{\varepsilon}$ on $\overline{w^{c} w^{d}}$ and $\overline{w^{b} w^{d}}$ is again $\Gamma_{3}$, we can repeat the construction in the triangle $T_{5}^{\varepsilon}$. Following the computation as in case 1 we get (4.4). This concludes the proof.

Proof of Theorem 4.1 We will suitably adapt the construction made in the proof of Proposition 4.4. By hypothesis the regions $E_{1}, E_{2}, E_{3}$ are enclosed by $C^{2}$-embedded curves $C_{i j}, i j \in\{12,23,31\}$, parametrized by arc length $c_{i j}:\left[0, r_{i j}\right] \rightarrow \mathbb{R}^{2}, i j \in\{12,23,31\}$. Moreover such curves meet $\partial D$ transversely and intersect each other (transversely) only at one point $Q$. Suppose that the angles formed at $Q$ by the three curves are all less than $\pi$ (the other case is similarly adapted from the corresponding case in the proof of Proposition 4.4). We will divide the domain $D$ into a finite number of subsets and define the sequence $\left\{u^{\varepsilon}\right\}$ on each of these sets.

Let $\delta_{\varepsilon}>0$ be such that $\delta_{\varepsilon} \rightarrow 0^{+}$as $\varepsilon \rightarrow 0^{+}$. Let $\tau \in\left[0, r_{i j}\right]$ be an arc length parameter on $C_{i j}$, with orthogonal coordinate $d$ that coincides with the signed distance from $C_{i j}$ negative in $E_{i}$ and positive in $E_{j}$. Let $Q_{i j} \in C_{i j}$ be the point with arc distance $\tau=\delta_{\varepsilon}$ from the origin $Q$. Consider the three lines normal to $C_{i j}$ at $Q_{i j}$. For $\delta_{\varepsilon}$ sufficiently small, since the angles at the origin are less than $\pi$ and the curves are of class $C^{2}$ up to the closure, these lines mutually meet at points $\zeta^{1}, \zeta^{2}$, and $\zeta^{3}$. Let $\varepsilon_{i j}$ be the length of $\overline{\zeta^{i} \zeta^{j}}$, which are of order $\varepsilon$. The tubular coordinates of the points $\zeta^{1}$ and $\zeta^{2}$ with respect to $C_{12}$ are $\left(d_{1}, \delta_{\varepsilon},\right)$ and $\left(d_{2}, \delta_{\varepsilon}\right)$, with $d_{2}-d_{1}=\varepsilon_{12}, d_{1}<0, d_{2}>0$. For $\delta_{\varepsilon}$ small enough we can consider the neighborhood of $C_{12}$ defined as

$$
S_{12}^{\varepsilon}:=\left\{(x, y) \in D: \tau(x, y) \geq \delta_{\varepsilon}, d(x, y) \in\left(d_{1}, d_{2}\right)\right\},
$$

where we have prolonged $C_{12}$ outside $D$ for convenience. Similarly we define $S_{23}^{\varepsilon}$ and $S_{31}^{\varepsilon}$. Let $T^{\varepsilon}$ be the triangle with vertices $\zeta^{1}, \zeta^{2}$, and $\zeta^{3}$.

Finally, let $E_{1}^{\varepsilon}, E_{2}^{\varepsilon}, E_{3}^{\varepsilon}$ be defined as in (4.6), and $u^{\varepsilon}$ as in (4.7).

Step 1 Definition of $u^{\varepsilon}$ on $S_{12}^{\varepsilon} \cup S_{23}^{\varepsilon} \cup S_{31}^{\varepsilon}$. We do the construction on $S_{12}^{\varepsilon}$, and $u^{\varepsilon}$ will be defined similarly on $S_{23}^{\varepsilon}$ and $S_{31}^{\varepsilon}$. We know that $c_{12}\left(\left[\delta_{\varepsilon}, r_{12}\right]\right)=C_{12} \cap S_{12}^{\varepsilon}$. The system of coordinates $(d, \tau)$ defines a $C^{1}$-diffeomorphism $h$ between the rectangle $\left[d_{1}, d_{2}\right] \times\left[\delta_{\varepsilon}, \rho_{12}\right]$ and its image $\mathcal{N}_{12}^{\varepsilon, \delta_{\varepsilon}}$ which contains $S_{12}^{\varepsilon}$, namely

$$
h:\left[d_{1}, d_{2}\right] \times\left[\delta_{\varepsilon}, \rho_{12}\right] \rightarrow \mathcal{N}_{12}^{\varepsilon, \delta_{\varepsilon}} ; \quad h(d, \tau):=c_{12}(\tau)+d \bar{\nu}(\tau),
$$

where $\bar{v}(\tau)$ is the unit normal vector pointing toward $E_{2}$ at $c_{12}(\tau)$ and $\rho_{12}=\rho_{12}^{\varepsilon} \geq r_{12}$ is the infimum of those $\rho$ for which $S_{12}^{\varepsilon} \subset \mathcal{N}_{12}^{\varepsilon, \delta_{\varepsilon}}$, see Fig. 8b.

Since $h$ is a $C^{1}$-diffeomorphism we have that

$$
h^{-1}: \mathcal{N}_{12}^{\varepsilon, \delta_{\varepsilon}} \rightarrow\left[d_{1}, d_{2}\right] \times\left[\delta_{\varepsilon}, \rho_{12}\right] ; \quad h^{-1}(x, y):=(d(x, y), \tau(x, y)),
$$

is the inverse of $h$ and is of class $C^{1}$. We want to estimate the Jacobian of $h^{-1}$. To this aim, we first see that $\nabla d\left(c_{12}(\tau)\right)=\bar{v}(\tau)$ since $c_{12}\left(\left[\delta_{\varepsilon}, \rho_{12}\right]\right)$ is the zero level set of $d$ and, from [2, Rem. 3(1)], we have

$$
\nabla d(h(d, \tau))=\bar{v}(\tau), \quad(d, \tau) \in\left[d_{1}, d_{2}\right] \times\left[\delta_{\varepsilon}, \rho_{12}\right] .
$$




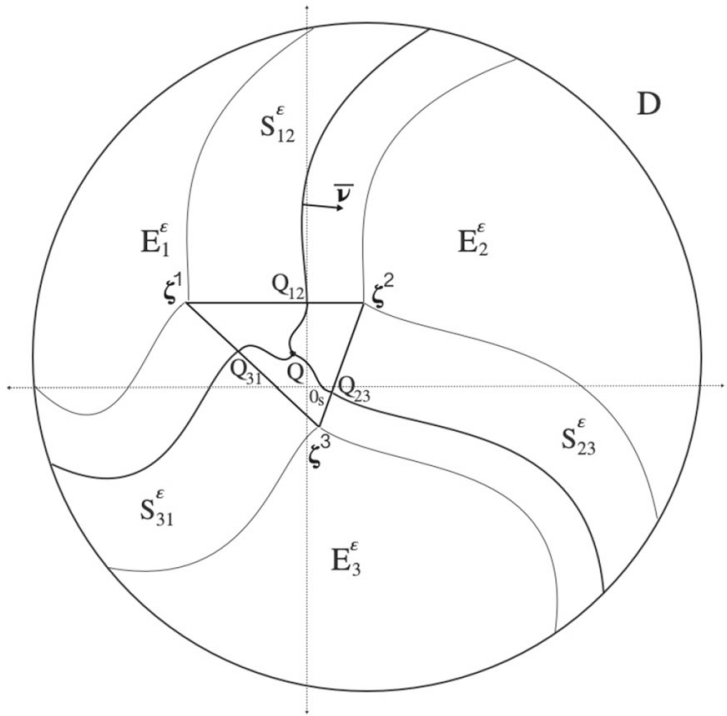

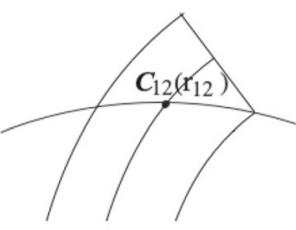

(b)

(a)

Fig. 8 Proof of Theorem 4.1

Fix $\tau \in\left[\delta_{\varepsilon}, \rho_{12}\right]$; by definition of tubular coordinates the segment $\left\{c_{12}(\tau)+d \bar{\nu}(\tau): d \in\right.$ $\left.\left[d_{1}, d_{2}\right]\right\}$ is a level set of the function $\tau(\cdot)$, hence

$$
\nabla \tau(x, y) \perp \bar{v}(\tau(x, y)),
$$

therefore

$$
\nabla \tau \cdot \nabla d=0 \quad \text { in } S_{12}^{\varepsilon} .
$$

Thus the Jacobian of $h^{-1}$ will be

$$
j\left(h^{-1}\right)=|\nabla \tau||\nabla d|=|\nabla \tau|,
$$

since $|\nabla d|=1$ in $\mathcal{N}_{12}^{\varepsilon, \delta_{\varepsilon}}$. Let us compute $\nabla \tau$; fix $d \in\left(d_{1}, d_{2}\right)$ and define $c_{12}^{d}(\tau):=c_{12}(\tau)+$ $d \bar{\nu}(\tau)$. Now recall (4.28) and that $\left(c_{12}^{d}\right)^{\prime}(\tau)$ is parallel to $\bar{\nu}^{\perp}(\tau)$, so that

$$
|\nabla \tau|=\nabla \tau \cdot \bar{v}^{\perp}=\frac{\nabla \tau \cdot\left(c_{12}^{d}\right)^{\prime}}{\left|\left(c_{12}^{d}\right)^{\prime}\right|} \quad \text { in } S_{12}^{\varepsilon} .
$$

Let us recall that $C_{12}$ is parametrized by arc length, i.e., $\left|c_{12}^{\prime}(\tau)\right|=1$, so that $\bar{v}^{\prime}(\tau)=$ $\left|c_{12}^{\prime \prime}(\tau)\right| c_{12}^{\prime}(\tau)$. Thus $\left(c_{12}^{d}\right)^{\prime}(\tau)=\left(1+d\left|c_{12}^{\prime \prime}(\tau)\right|\right) c_{12}^{\prime}(\tau)$. Since $\tau \circ c_{12}^{d}=$ Id it follows that $\nabla \tau\left(c_{12}^{d}(\tau)\right)^{T}\left(c_{12}^{d}\right)^{\prime}(\tau)=\nabla \tau\left(c_{12}^{d}(\tau)\right) \cdot\left(c_{12}^{d}\right)^{\prime}(\tau)=1$. Therefore, from (4.31), we deduce

$$
|\nabla \tau|=\frac{1}{1+d\left|c_{12}^{\prime \prime}(\tau)\right|} \quad \text { in } S_{12}^{\varepsilon},
$$

and in particular $\lim _{d \rightarrow 0}|\nabla \tau|=1$ uniformly in $S_{12}^{\varepsilon}$.

We are ready to define $u^{\varepsilon}$ in $S_{12}^{\varepsilon}$. We first set $\psi_{12}^{\varepsilon}$ as in (4.9) with $r_{12}+c_{\varepsilon}=\rho_{12}$, i.e., $\psi_{12}^{\varepsilon}(\tau)=\bar{\kappa}_{\varepsilon}\left(z-\delta_{\varepsilon}\right)$, setting $\bar{\kappa}_{\varepsilon}:=\frac{\rho_{12}}{\rho_{12}-\delta_{\varepsilon}}$. Then we define $\tilde{u}^{\varepsilon}$ on $\left[d_{1}, d_{2}\right] \times\left[\delta_{\varepsilon}, \rho_{12}\right]$ as in the right hand side of (4.11) and set 


$$
u^{\varepsilon}:=\tilde{u}^{\varepsilon} \circ h^{-1} \quad \text { in } S_{12}^{\varepsilon} .
$$

Explicitly, recalling that $\xi=\frac{\alpha_{2}-\alpha_{1}}{\ell_{12}}$ and $\eta=\xi^{\perp}$, for $(x, y) \in S_{12}^{\varepsilon}$ we have

$$
\begin{aligned}
u^{\varepsilon}(x, y):= & \alpha_{1}+\left(\frac{d(x, y)-d_{1}}{\varepsilon_{12}}\right) \ell_{12} \xi \\
& +m_{12}^{\sigma}\left(\frac{d(x, y)-d_{1}}{\varepsilon_{12}} \ell_{12}, \bar{\kappa}_{\varepsilon}\left(\tau(x, y)-\delta_{\varepsilon}\right)\right) \eta .
\end{aligned}
$$

Observe that $u^{\varepsilon}=\left(u_{1}^{\varepsilon}, u_{2}^{\varepsilon}\right) \in \operatorname{Lip}\left(S_{12}^{\varepsilon} ; \mathbb{R}^{2}\right), u^{\varepsilon}=\alpha_{1}$ on $\left\{(x, y) \in S_{12}^{\varepsilon}: d(x, y)=d_{1}\right\}, u^{\varepsilon}=$ $\alpha_{2}$ on $\left\{(x, y) \in S_{12}^{\varepsilon}: d(x, y)=d_{2}\right\}$, and by construction there exists $w_{a} \in h\left(\left[d_{1}, d_{2}\right] \times\left\{\delta_{\varepsilon}\right\}\right)$ such that $u^{\varepsilon}\left(w_{a}\right)=0_{T}$. Write for simplicity $\tilde{m}=m_{12}^{\sigma}$. We have

$$
\begin{aligned}
& \nabla u_{1}^{\varepsilon}=\left(\frac{\ell_{12} \xi^{1}}{\varepsilon_{12}} d_{x}+\frac{\ell_{12} \eta^{1}}{\varepsilon_{12}} \tilde{m}_{s} d_{x}+\bar{\kappa}_{\varepsilon} \tilde{m}_{t} \tau_{x} \eta^{1}, \frac{\ell_{12} \xi^{1}}{\varepsilon_{12}} d_{y}+\frac{\ell_{12} \eta^{1}}{\varepsilon_{12}} \tilde{m}_{s} d_{y}+\bar{\kappa}_{\varepsilon} \tilde{m}_{t} \tau_{y} \eta^{1}\right), \\
& \nabla u_{2}^{\varepsilon}=\left(\frac{\ell_{12} \xi^{2}}{\varepsilon_{12}} d_{x}+\frac{\ell_{12} \eta^{2}}{\varepsilon_{12}} \tilde{m}_{s} d_{x}+\bar{\kappa}_{\varepsilon} \tilde{m}_{t} \tau_{x} \eta^{2}, \frac{\ell_{12} \xi^{2}}{\varepsilon_{12}} d_{y}+\frac{\ell_{12} \eta^{2}}{\varepsilon_{12}} \tilde{m}_{s} d_{y}+\bar{\kappa}_{\varepsilon} \tilde{m}_{t} \tau_{y} \eta^{2}\right),
\end{aligned}
$$

where $\tilde{m}_{s}, \tilde{m}_{t}$ denote the partial derivatives of $\tilde{m}$ with respect to $s=\frac{d(x, y)-d_{1}}{\varepsilon_{12}} \ell_{12}$ and $t=\bar{\kappa}_{\varepsilon}\left(\tau(x, y)-\delta_{\varepsilon}\right)$, respectively, and are evaluated at $\left(\frac{d(x, y)-d_{1}}{\varepsilon_{12}} \ell_{12}, \bar{\kappa}_{\varepsilon}\left(\tau(x, y)-\delta_{\varepsilon}\right)\right)$. Hence

$$
\begin{aligned}
\left|\nabla u_{1}^{\varepsilon}\right|^{2}+\left|\nabla u_{2}^{\varepsilon}\right|^{2}= & \frac{\ell_{12}^{2}}{\varepsilon_{12}^{2}}|\nabla d|^{2}+\frac{\ell_{12}^{2}}{\varepsilon_{12}^{2}}|\nabla d|^{2}\left(\tilde{m}_{s}\right)^{2}+\bar{\kappa}_{\varepsilon}^{2}|\nabla \tau|^{2}\left(\tilde{m}_{t}\right)^{2} \\
& +\frac{2 \ell_{12}}{\varepsilon_{12}} \bar{\kappa}_{\varepsilon}(\nabla d \cdot \nabla \tau) \tilde{m}_{s} \tilde{m}_{t}
\end{aligned}
$$

where we have used $|\xi|=|\eta|=1$ and $\xi_{1} \eta_{1}+\xi_{2} \eta_{2}=0$. From (4.29) we have

$$
\left|\nabla u_{1}^{\varepsilon}\right|^{2}+\left|\nabla u_{2}^{\varepsilon}\right|^{2}=\frac{\ell_{12}^{2}}{\varepsilon_{12}^{2}}+\frac{\ell_{12}^{2}}{\varepsilon_{12}^{2}}\left(\tilde{m}_{s}\right)^{2}+\bar{\kappa}_{\varepsilon}^{2}|\nabla \tau|^{2}\left(\tilde{m}_{t}\right)^{2} \text {. }
$$

Moreover

$$
\begin{aligned}
\left(\frac{\partial u_{1}^{\varepsilon}}{\partial x} \frac{\partial u_{2}^{\varepsilon}}{\partial y}-\frac{\partial u_{1}^{\varepsilon}}{\partial y} \frac{\partial u_{2}^{\varepsilon}}{\partial x}\right)^{2} & =\frac{\ell_{12}^{2}}{\varepsilon_{12}^{2}} \bar{\kappa}_{\varepsilon}^{2}\left(\widetilde{m}_{t}\right)^{2}\left(d_{x} \tau_{y}-d_{y} \tau_{x}\right)^{2}\left(\xi^{1} \eta^{2}-\xi^{2} \eta^{1}\right)^{2} \\
& =\frac{\ell_{12}^{2}}{\varepsilon_{12}^{2}} \bar{\kappa}_{\varepsilon}^{2}\left(\widetilde{m}_{t}\right)^{2}|\nabla \tau|^{2}
\end{aligned}
$$

where again $\tilde{m}_{s}, \tilde{m}_{t}$ are evaluated at $\left(\frac{d(x, y)-d_{1}}{\varepsilon_{12}} \ell_{12}, \bar{\kappa}_{\varepsilon}\left(\tau(x, y)-\delta_{\varepsilon}\right)\right)$, and we have used (4.29), (4.30), and $\xi^{1} \eta^{2}-\xi^{2} \eta^{1}=1$. Therefore from (4.35) and (4.36) we obtain

$$
\begin{aligned}
1 & +\left|\nabla u_{1}^{\varepsilon}\right|^{2}+\left|\nabla u_{2}^{\varepsilon}\right|^{2}+\left(\frac{\partial u_{1}^{\varepsilon}}{\partial x} \frac{\partial u_{2}^{\varepsilon}}{\partial y}-\frac{\partial u_{1}^{\varepsilon}}{\partial y} \frac{\partial u_{2}^{\varepsilon}}{\partial x}\right)^{2} \\
& =1+\frac{\ell_{12}^{2}}{\varepsilon_{12}^{2}}\left(1+\left(\tilde{m}_{s}\right)^{2}+\left(\tilde{m}_{t}\right)^{2} \bar{\kappa}_{\varepsilon}^{2}\left(1+\frac{\varepsilon_{12}^{2}}{\ell_{12}^{2}}\right)|\nabla \tau|^{2}\right) .
\end{aligned}
$$


As a consequence

$$
\begin{aligned}
& \mathbb{A}\left(u^{\varepsilon}, S_{12}^{\varepsilon}\right)=\frac{\ell_{12}}{\varepsilon_{12}} \int_{S_{12}^{\varepsilon}} \sqrt{1+\left(\tilde{m}_{s}\right)^{2}+\left(\tilde{m}_{t}\right)^{2} \bar{\kappa}_{\varepsilon}^{2}\left(1+\frac{\varepsilon_{12}^{2}}{\ell_{12}^{2}}\right)|\nabla \tau|^{2}+O\left(\varepsilon^{2}\right)} \mathrm{d} x \mathrm{~d} y \\
= & \frac{1}{\bar{\kappa}_{\varepsilon}} \int_{\mathrm{R}_{12} \backslash P_{\varepsilon}} \frac{1}{|\nabla \tau|} \sqrt{1+\left(\tilde{m}_{s}(s, t)\right)^{2}+\left(\tilde{m}_{t}(s, t)\right)^{2} \bar{\kappa}_{\varepsilon}^{2}\left(1+\frac{\varepsilon_{12}^{2}}{\ell_{12}^{2}}\right)|\nabla \tau|^{2}+O\left(\varepsilon^{2}\right)} \mathrm{d} s \mathrm{~d} t,
\end{aligned}
$$

where $\tilde{m}_{s}, \tilde{m}_{t}$ in the first integral are evaluated at $\left(\frac{d(x, y)-d_{1}}{\varepsilon_{12}} \ell_{12}, \bar{\kappa}_{\varepsilon}\left(\tau(x, y)-\delta_{\varepsilon}\right)\right), \nabla \tau$ in the second integral is evaluated at $(x, y)=\Phi^{-1}(s, t)$ and the last equality follows from the change of variables

$$
\Phi:(x, y) \in \mathcal{N}_{12}^{\varepsilon} \rightarrow\left(\frac{d(x, y)-d_{1}}{\varepsilon_{12}} \ell_{12}, \bar{\kappa}_{\varepsilon}\left(\tau(x, y)-\delta_{\varepsilon}\right)\right)=(s, t) \in \mathrm{R}_{12},
$$

and $P_{\varepsilon}:=\mathrm{R}_{12} \backslash \Phi\left(S_{12}^{\varepsilon}\right)$ (see Fig. 5). Here one checks that $\Phi=H \circ h^{-1}$ with $H(d, \tau)=$ $\left(\frac{d-d_{1}}{\varepsilon_{12}} \ell_{12}, \bar{\kappa}_{\varepsilon}\left(\tau-\delta_{\varepsilon}\right)\right)$ so that, using (4.30), the Jacobian of the change of variable is $\frac{1}{\left|\nabla \tau\left(\Phi^{-1}(s, t)\right)\right|} \frac{\varepsilon_{12}}{\ell_{12} \bar{\kappa}_{\varepsilon}}$. Hence, recalling (4.32) and that $\bar{\kappa}_{\varepsilon} \rightarrow 1$ as $\varepsilon \rightarrow 0^{+}$,

$$
\lim _{\varepsilon \rightarrow 0^{+}} \mathbb{A}\left(u^{\varepsilon}, S_{12}^{\varepsilon}\right)=\int_{\mathrm{R}_{12}} \sqrt{1+\left(\widetilde{m}_{s}\right)^{2}+\left(\tilde{m}_{t}\right)^{2}} \mathrm{~d} s \mathrm{~d} t .
$$

Now, let us recall that $\tilde{m}=m_{12}^{\sigma}$ is the approximating function as in (4.3); it follows that

$$
\int_{\mathrm{R}_{12}} \sqrt{1+\left(\widetilde{m}_{s}\right)^{2}+\left(\widetilde{m}_{t}\right)^{2}} \mathrm{~d} s \mathrm{~d} t=\mathfrak{A}_{12}(\Gamma)+O(\sigma) .
$$

Hence, employing the same construction in the strips $S_{23}^{\varepsilon}$ and $S_{31}^{\varepsilon}$, and using (4.39) we obtain from a diagonal argument with $\sigma=\sigma_{\varepsilon} \rightarrow 0$ as $\varepsilon \rightarrow 0^{+}$,

$$
\lim _{\varepsilon \rightarrow 0^{+}} \mathbb{A}\left(u^{\varepsilon}, S_{23}^{\varepsilon} \cup S_{31}^{\varepsilon} \cup S_{23}^{\varepsilon}\right)=\mathfrak{A}_{12}(\Gamma)+\mathfrak{A}_{23}(\Gamma)+\mathfrak{A}_{31}(\Gamma) .
$$

Step 2 Definition of $u^{\varepsilon}$ on $T^{\varepsilon}$. This is identical to Step 3 of the proof of Proposition 4.4 and therefore $\left\{u^{\varepsilon}\right\} \subset \operatorname{Lip}\left(B_{r} ; \mathbb{R}^{2}\right)$ and (4.23) holds. Following the same computations of Proposition 4.4 the conclusion follows.

Step 3 For the case where two of the curves $C_{i j}, i j \in\{12,23,31\}$ meet at $Q$ with an angle larger than or equal to $\pi$ we replace $T^{\varepsilon}$ with $H^{\varepsilon}$ defined in case 2 of Proposition 4.4, in the above construction.

\section{Existence of minimizers for the functional $\mathcal{G}$}

Let $D$ be an open disk centered at the origin such that $E_{1}, E_{2}, E_{3}$ are circular sectors with $120^{\circ}$ angles and let $\mathrm{T}$ be an equilateral triangle. Let $p$ be the barycenter of T and $\widetilde{\Gamma}_{i}$ be the segment connecting $\alpha_{i}$ and $p, i \in\{1,2,3\}$. Hence $\widetilde{\Gamma}=\left(\widetilde{\Gamma}_{1}, \widetilde{\Gamma}_{2}, \widetilde{\Gamma}_{3}\right) \in X_{\text {Lip }}$ so that

$$
\inf \left\{\mathcal{G}(\Gamma): \Gamma \in X_{\text {Lip }}\right\} \leq \mathcal{G}(\widetilde{\Gamma}) .
$$

Moreover we have

$$
|D|+\mathcal{G}(\widetilde{\Gamma})=\mathcal{A}(u, D) \leq|D|+\inf \left\{\mathcal{G}(\Gamma): \Gamma \in X_{\mathrm{Lip}}\right\},
$$


where $u=u_{\text {symm }}$ (see Sect. 1), and the equality follows from [12, Section 3] and the inequality follows from Proposition 4.4. Thus

$$
\mathcal{G}(\widetilde{\Gamma})=\min \left\{\mathcal{G}(\Gamma): \Gamma \in X_{\text {Lip }}\right\} .
$$

Hence in this symmetric situation the optimal connection is obtained through the Steiner graph connecting $\alpha_{1}, \alpha_{2}$ and $\alpha_{3}$. This motivates the analysis of this section, which is carried on without symmetry assumptions.

We recall that given a connection $\Gamma=\left(\Gamma_{1}, \Gamma_{2}, \Gamma_{3}\right) \in X$ we denote by $\varphi_{i j}=\varphi_{i j}\left(\Gamma_{i j}\right)$ : $\left[0, \ell_{i j}\right] \rightarrow[0$, diamT $]$ the function whose graph is $\Gamma_{i j}=\Gamma_{i} \cup \Gamma_{j}$ (see (3.2)).

Definition 5.1 (Convergence in $X$ ) We say that a sequence $\left\{\Gamma^{n}\right\} \subset X$ converges to $\Gamma \in X$ in $X$, and we write $\Gamma^{n} \rightarrow \Gamma$ in $X$, if

$$
\varphi_{i j}\left(\Gamma_{i j}^{n}\right) \rightarrow \varphi_{i j}\left(\Gamma_{i j}\right) \quad \text { in } L^{1}\left(\left[0, \ell_{i j}\right]\right), \quad i j \in\{12,23,31\} .
$$

\subsection{Density and approximation}

We start to show that a BV connection $\Gamma \in X$ can be approximated by Lipschitz connections; the difficulty is to keep graphicality of each branch of the approximating connections with respect to the two corresponding edges of $\mathrm{T}$ at the same time.

Recall that $\Gamma_{i}$ is the branch of the connection $\Gamma$ connecting $\alpha_{i}$ to $p$ and that by Definition 3.1 we have

$$
\Gamma_{i}\left\llcorner\mathrm{~T} \backslash \overline{p \pi_{i j}(p)} \cup \overline{p \pi_{k i}(p)}=\Gamma_{\varphi_{i j}\left\llcorner\left[0, w_{i j}\right)\right.}=\Gamma_{\varphi_{k i}\left\llcorner\left(w_{k i}, \ell_{k i}\right]\right.} .\right.
$$

Note that we excluded the vertical parts over the points $\pi_{i j}(p), i j \in\{12,23,31\}$, due to Remark 3.2; however, we still have

$$
\Gamma_{i} \cup \Gamma_{j}=\Gamma_{\varphi_{i j}}
$$

Lemma 5.2 (Piecewise linear approximation) For any $\Gamma \in X$ with target triple point $p \in \mathrm{T}$ there exists a sequence $\left\{\Gamma^{n}\right\} \subset X_{\text {Lip }}$ of connections with target triple point $p$ such that $\varphi_{i j}\left(\Gamma_{i j}^{n}\right), i j \in\{12,23,31\}$, is a piecewise linear ${ }^{1}$ function,

$$
\mathcal{H}^{1}\left(\Gamma_{i j}^{n}\right) \leq \mathcal{H}^{1}\left(\Gamma_{i j}\right)
$$

and

$$
\Gamma^{n} \rightarrow \Gamma \text { in } X
$$

Proof Let $i j=12$ and let $w_{12}$ be defined as in (3.1). Let $n_{12}:=(0,1) \in \mathbb{R}^{2}$ be the inward unit normal to $\overline{\alpha_{1} \alpha_{2}}, n_{31}:=(\alpha, \beta)$ be the inward unit normal to $\overline{\alpha_{3} \alpha_{1}}$, and $v(\bar{s}):=\left(v^{1}(\bar{s}), v^{2}(\bar{s})\right)$ be the generalized outward unit normal at the point $\left(\bar{s}, \varphi_{12}(\bar{s})\right)$ to the generalized graph $\Gamma_{\varphi_{12}\left\llcorner\left[0, w_{12}\right]\right.}$ of $\varphi_{12}\left\llcorner\left[0, w_{12}\right]\right.$ (for all $\bar{s}$ where it exists), see Fig. 9. Without loss of generality, we may assume $\Gamma_{1}=\Gamma_{\varphi_{12}} L_{\left[0, w_{12}\right]}$. We start to show that $\varphi_{12}$ cannot have too negative slope, otherwise $\Gamma_{1}$ loses graphicality with respect to $\overline{\alpha_{3} \alpha_{1}}$.

Step 1 We claim that

$$
\varphi_{12}^{\prime}\left\llcorner\left[0, w_{12}\right] \geq \frac{\beta}{\alpha},\right.
$$

\footnotetext{
1 This means that it is Lipschitz piecewise linear with at most finitely many points of nondifferentiability.
} 


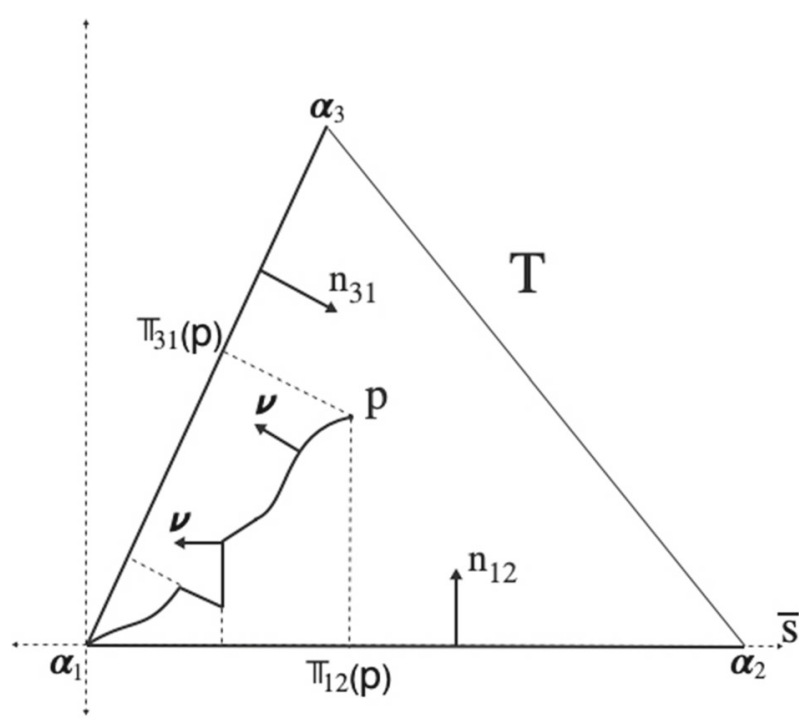

Fig. 9 Proof of Lemma 5.2. For convenience we choose $\alpha_{1}$ to be the origin

in the sense of measures, i.e.,

$$
\varphi_{12}^{\prime}(B) \geq \frac{\beta}{\alpha} \mathcal{L}^{1}(B), \quad \forall B \subseteq\left[0, w_{12}\right] \text { Borel set. }
$$

From the graphicality with respect to $\overline{\pi_{31}(p) \alpha_{1}}$ we have, for all $\bar{s}$ where $v(\bar{s})$ exists,

$$
v(\bar{s}) \cdot n_{31} \leq 0 .
$$

Set

$$
\begin{aligned}
& I^{r}:=\left\{\bar{s} \in\left[0, w_{12}\right]: v(\bar{s}) \text { is defined, and } v^{2}(\bar{s})>0\right\}, \\
& I^{s}:=\left\{\bar{s} \in\left[0, w_{12}\right]: v(\bar{s}) \text { is defined, and } v^{2}(\bar{s})=0\right\}
\end{aligned}
$$

note that $v(\bar{s})=\frac{1}{\sqrt{1+\left(\dot{\varphi}_{12}(\bar{s})\right)^{2}}}\left(-\dot{\varphi}_{12}(\bar{s}), 1\right)$ for any $\bar{s} \in I^{r}$. From $[10$, Thm. 7 p. 301 and Thm. 5 p. 379], we have

$$
\dot{\varphi}_{12} d \bar{s}=\varphi_{12}^{\prime}\left\llcorner I^{r}, \quad \dot{\varphi}_{12}^{(j)}+\dot{\varphi}_{12}^{(c)}=\varphi_{12}^{\prime}\left\llcorner I^{s} .\right.\right.
$$

From (5.4) it follows that

$$
v(\bar{s})=(-1,0) \quad \forall \bar{s} \in I^{s} \quad \text { and } \quad \dot{\varphi}_{12}(\bar{s}) \geq \frac{\beta}{\alpha} \quad \forall \bar{s} \in I^{r} .
$$

From [10, Thm. 4, p. 378] we have

$$
\dot{\varphi}_{12}^{(j)}+\dot{\varphi}_{12}^{(c)}=-v^{1}|\mu|\left\llcorner I^{s}=|\mu|\left\llcorner I^{s},\right.\right.
$$

where $\mu:=\left(\varphi_{12}^{\prime},-\mathcal{L}^{1}\right)=\left(-v^{1},-v^{2}\right)|\mu|$ and the second equality follows from the first formula in (5.6).

For any Borel set $B \subseteq\left[0, w_{12}\right]$ we deduce

$$
\varphi_{12}^{\prime}(B)=\int_{B} \dot{\varphi}_{12} d \bar{s}+\dot{\varphi}_{12}^{(j)}(B)+\dot{\varphi}_{12}^{(c)}(B) \geq \frac{\beta}{\alpha} \mathcal{L}^{1}(B)+|\mu| L I^{s}(B) \geq \frac{\beta}{\alpha} \mathcal{L}^{1}(B) .
$$


Step 2 Given $\epsilon \in(0,1)$, we choose $n=n(\epsilon) \in \mathbb{N}$ and points

$$
\xi_{0}=0<\xi_{1}<\cdots<\xi_{n-1}<\xi_{n}=w_{12},
$$

such that each $\xi_{i}, i \in\{1, \ldots, n-1\}$, is a point of continuity of $\varphi_{12}$, and if we define $\varphi^{n} \in \operatorname{Lip}\left(\left[0, w_{12}\right]\right)$ as the piecewise linear interpolation with

$$
\varphi^{n}\left(\xi_{i}\right)=\varphi_{12}\left(\xi_{i}\right), \quad i=0, \ldots, n,
$$

then, by taking $n$ large enough,

$$
\left\|\varphi^{n}-\varphi_{12}\right\|_{L^{1}\left(\left(0, w_{12}\right)\right)}<\epsilon
$$

The graph of $\varphi^{n}$ may still have vertical parts over $\overline{\pi_{31}(p) \alpha_{1}}$. Indeed from [3, Theorem 3.30], and the fact that $\xi_{i}$ are continuity points of $\varphi_{12}$ we have

$$
\varphi^{n^{\prime}}(\xi)=\frac{\varphi_{12}\left(\xi_{i}\right)-\varphi_{12}\left(\xi_{i-1}\right)}{\xi_{i}-\xi_{i-1}}=\frac{\varphi_{12}^{\prime}\left(\left(\xi_{i-1}, \xi_{i}\right)\right)}{\xi_{i}-\xi_{i-1}} \geq \frac{\beta}{\alpha}, \quad \xi \in\left(\xi_{i-1}, \xi_{i}\right),
$$

and equality may hold; hence, the graph of $\varphi^{n}$ over $\overline{\pi_{31}(p) \alpha_{1}}$ may have finitely many vertical parts. It is now sufficient to repeat the argument with $\varphi^{n}$ in place of $\varphi_{12}$, choosing a suitable partition of $\left[w_{31}, \ell_{31}\right]$, so to ensure that (recall that $\varphi^{n \prime}$ exists out of finitely many points)

$$
\varphi^{n \prime}>\frac{\beta}{\alpha} \text { at the differentiability points of }\left[0, w_{12}\right] .
$$

In this way $\varphi^{n}$ is a Lipschitz graph also with respect to $\overline{\pi_{31}(p) \alpha_{1}}$.

Step 3 We have

$$
\begin{aligned}
& \mathcal{H}^{1}\left(\Gamma_{\varphi^{n}}\right)=\sum_{i=1}^{n} \int_{\xi_{i-1}}^{\xi_{i}} \sqrt{1+\left|\varphi^{n \prime}(s)\right|^{2}} \mathrm{~d} s=\sum_{i=1}^{n}\left|\left(\xi_{i}, \varphi^{n}\left(\xi_{i}\right)\right)-\left(\xi_{i-1}, \varphi^{n}\left(\xi_{i-1}\right)\right)\right| \\
& \leq \sup \left\{\sum_{i=1}^{m}\left|\left(\eta_{i}, \varphi_{12}\left(\eta_{i}\right)\right)-\left(\eta_{i-1}, \varphi_{12}\left(\eta_{i-1}\right)\right)\right|: m \in \mathbb{N}, \eta_{0}=0<\eta_{1}<\right. \\
& \left.\quad \cdots<\eta_{m-1}<\eta_{m}=w_{12}\right\} \\
& =\int_{\left[0, w_{12}\right]}\left|\Phi_{12}^{\prime}\right|=\mathcal{H}^{1}\left(\Gamma_{\varphi_{12}} L_{\left[0, w_{12}\right]}\right),
\end{aligned}
$$

where $\Phi_{12} \in \mathrm{BV}\left(\left[0, w_{12}\right] ; \mathbb{R}^{2}\right)$ is defined as $\Phi_{12}(\xi):=\left(\xi, \varphi_{12}(\xi)\right)$, and the last equality follows from [3, (3.24), p.136], and the fact that $\varphi_{12}$ is a good representative.

Step 4 Define

$$
\Gamma_{1}^{n}:=\Gamma_{\varphi^{n}} .
$$

Similarly we define $\Gamma_{2}^{n}$ and $\Gamma_{3}^{n}$, and we set $\Gamma_{i j}^{n}:=\Gamma_{i}^{n} \cup \Gamma_{j}^{n}$. Then $\Gamma^{n}:=\left(\Gamma_{1}^{n}, \Gamma_{2}^{n}, \Gamma_{3}^{n}\right)$ satisfies the required properties.

Proposition 5.3 (Uniform estimate of the length) There exists $c>0$ depends on $\mathrm{T}$ such that for all $\Gamma \in X$ we have

$$
\mathcal{H}^{1}\left(\Gamma_{i j}\right) \leq c, \quad i j \in\{12,23,31\} .
$$


Proof Let $\Gamma \in X$ be a connection through $p \in \mathrm{T}$. Without loss of generality, we may assume that $p \neq \alpha_{1}$. From (5.8) we have

$$
\begin{aligned}
\mathcal{H}^{1}\left(\Gamma_{1}\right)= & \sup \left\{\sum_{i=1}^{m}\left|\left(\eta_{i}, \varphi_{12}\left(\eta_{i}\right)\right)-\left(\eta_{i-1}, \varphi_{12}\left(\eta_{i-1}\right)\right)\right|: m \in \mathbb{N}, \eta_{0}=0<\eta_{1}\right. \\
& \left.<\cdots<\eta_{m-1}<\eta_{m}=w_{12}\right\} .
\end{aligned}
$$

Choose a partition

$$
\xi_{0}=0<\xi_{1}<\cdots<\xi_{h-1}<\xi_{h}=w_{12} .
$$

Let $\Gamma_{1}^{h}$ be the piecewise linear interpolation connecting $\left(\xi_{i-1}, \varphi_{12}\left(\xi_{i-1}\right)\right)$ and $\left(\xi_{i}, \varphi_{12}\left(\xi_{i}\right)\right)$, $i \in\{1, \ldots, h\}$. The unit tangent to $\Gamma_{1}^{h}$ is enclosed in the angle formed by $n_{12}$ and $n_{31}$, the unit normals to $\overline{\alpha_{1} \alpha_{2}}$ and $\overline{\alpha_{3} \alpha_{1}}$ (due to the graphicality condition with respect to $\overline{\alpha_{1} \alpha_{2}}$ and $\overline{\alpha_{3} \alpha_{1}}$ ), see Fig. 10. It follows that $\Gamma_{1}^{h}$ is the graph of a function $\phi_{12}^{h}$ over the segment $\overline{\alpha_{1} p}$. Fix a Cartesian coordinate system in which the $t$-axis is the line $\overline{\alpha_{1} p}$ and the origin is $\alpha_{1}$.

For any $t \in\left[0,\left|\alpha_{1}-p\right|\right]$ (up to a finite set) let $\tau(t)$ be the unit tangent to $\Gamma_{1}^{h}$ at $\left(t, \phi_{12}^{h}(t)\right.$ ) and let $n=(1,0)$ and $n^{\perp}=(0,1)$. Hence $\phi_{12}^{h}{ }^{\prime}=\frac{\tau \cdot n^{\perp}}{\tau \cdot n}$ satisfies

$$
c_{1}^{-}:=\frac{n_{31} \cdot n^{\perp}}{n_{31} \cdot n} \leq \phi_{12}^{h^{\prime}} \leq \frac{n_{12} \cdot n^{\perp}}{n_{12} \cdot n}=: c_{1}^{+} .
$$

Note that one between $\left|c_{1}^{-}\right|$and $\left|c_{1}^{+}\right|$might be $+\infty$, since one of the sides $\overline{\alpha_{1} \alpha_{2}}$ or $\overline{\alpha_{3} \alpha_{1}}$ can be horizontal (this happens only if the point $p$ is on one side of the triangle). However, we always have $c_{1}^{-} \leq 0, c_{1}^{+} \geq 0$. Furthermore, when the angle $\hat{\alpha}_{1}$ in $\alpha_{1}$ is less than or equal to $\frac{\pi}{2}$, it follows that $\tilde{c}_{1}:=\min \left\{\left|c_{1}^{-}\right|,\left|c_{1}^{+}\right|\right\} \leq\left|\tan \left(\frac{\pi}{2}-\frac{\hat{\alpha}_{1}}{2}\right)\right|$. In the case $\hat{\alpha}_{1}>\frac{\pi}{2}$, using that $p \in T_{\text {int }}$, we have $\max \left\{\left|c_{1}^{-}\right|,\left|c_{1}^{+}\right|\right\} \leq\left|\tan \left(\pi-\hat{\alpha}_{1}\right)\right|$. Thus the only difficulty to prove is that the length of $\Gamma_{1}^{h}$ is controlled when $\hat{\alpha}_{1} \leq \frac{\pi}{2}$. So let us assume this and in addition that $\left|c_{1}^{-}\right|=\tilde{c}_{1}$ (the other case is similar). Since $\phi_{12}^{h}\left(\left|\alpha_{1}-p\right|\right)=\phi_{12}^{h}(0)=0$ we have

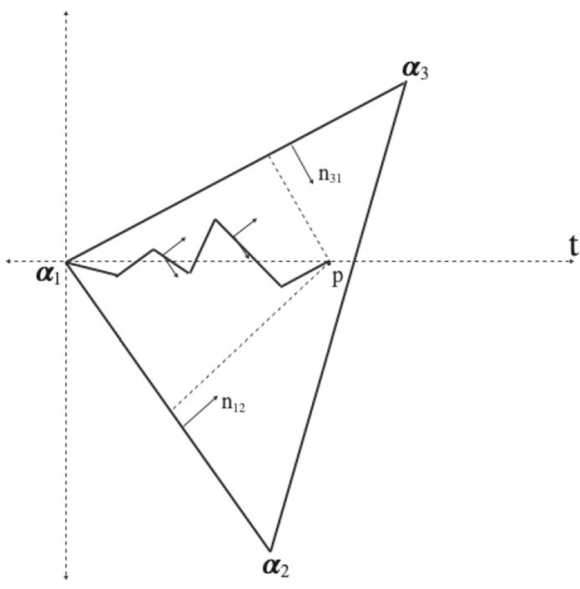

(a)

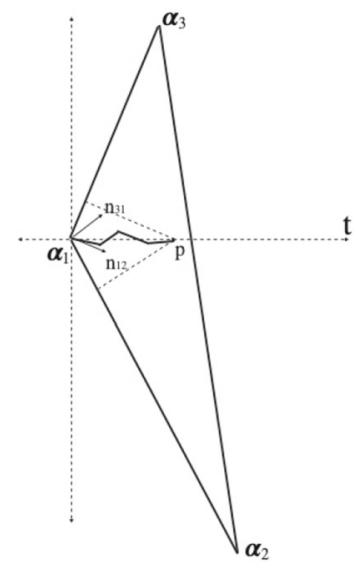

(b)

Fig. 10 a T with angles less than or equal to $\frac{\pi}{2}$ and $\mathbf{b} \mathrm{T}$ with an angle greater than $\frac{\pi}{2}$ 


$$
0=\phi_{12}^{h}{ }^{\prime}\left(\left[0,\left|\alpha_{1}-p\right|\right]\right)=\left(\phi_{12}^{h}\right)^{+}\left(\left[0,\left|\alpha_{1}-p\right|\right]\right)-\left(\phi_{12}^{h{ }^{\prime}}\right)^{-}\left(\left[0,\left|\alpha_{1}-p\right|\right]\right),
$$

where $\left(\phi_{12}^{h}\right)^{+}$and $\left(\phi_{12}^{h}{ }^{\prime}\right)^{-}$are the positive and negative parts of the measure $\phi_{12}^{h}{ }^{\prime}=\dot{\phi}_{12}^{h} \mathrm{~d} t$. Thus we estimate

$$
\begin{aligned}
\left|\mathcal{H}^{1}\left(\Gamma_{1}^{h}\right)\right| & =\int_{0}^{\left|\alpha_{1}-p\right|} \sqrt{1+\dot{\phi}_{12}^{h}(t)^{2}} \mathrm{~d} t \\
& \leq\left|\alpha_{1}-p\right|+\left|\phi_{12}^{h^{\prime}}\right|\left(\left[0,\left|\alpha_{1}-p\right|\right]\right)=\left|\alpha_{1}-p\right|+2\left(\phi_{12}^{h^{\prime}}\right)^{-}\left(\left[0,\left|\alpha_{1}-p\right|\right]\right) \\
& \leq\left|\alpha_{1}-p\right|+2\left|\alpha_{1}-p\right| \tilde{c}_{1}=: c_{1} .
\end{aligned}
$$

Then $c_{1}$ is a positive constant depending only on the geometry of T.

From (5.11) and (5.10), it then follows

$$
\mathcal{H}^{1}\left(\Gamma_{1}\right) \leq c_{1} .
$$

Similarly we may show that $\mathcal{H}^{1}\left(\Gamma_{2}\right) \leq c_{2}$ and $\mathcal{H}^{1}\left(\Gamma_{3}\right) \leq c_{3}$ for $c_{2}, c_{3}>0$ depending only on $\mathrm{T}$. This proves (5.9) with $c=c_{1}+c_{2}+c_{3}$.

The next lemma shows continuity of the sum of the three areas of area minimizing surfaces defining $\mathcal{G}$ in (3.9), with respect to the $L^{1}$ convergence of the traces in $\mathrm{T}$.

Proposition 5.4 (Continuity of $\mathcal{G}$ ) Let $\Gamma \in X$, and let $\left\{\Gamma^{n}\right\} \subset X$ be a sequence converging to $\Gamma$ in $X$. Then

$$
\lim _{n \rightarrow+\infty} \mathcal{G}\left(\Gamma^{n}\right)=\mathcal{G}(\Gamma) .
$$

Proof Since $\Gamma \in X$ and $\left\{\Gamma^{n}\right\} \subset X$ we have $\varphi_{i j} \in \mathrm{BV}\left(\left[0, \ell_{i j}\right]\right)$ and $\left\{\varphi_{i j}^{n}\right\} \subset \mathrm{BV}\left(\left[0, \ell_{i j}\right]\right)$, where $\varphi_{i j}:=\varphi_{i j}\left(\Gamma_{i j}\right), \varphi_{i j}^{n}:=\varphi_{i j}\left(\Gamma_{i j}^{n}\right)$.

Hence from (1.6) and Sect. 3.1 it follows that there exist $\widehat{m}_{i j}, \widehat{m}_{i j}^{n} \in W^{1,1}\left(\widehat{\mathrm{R}}_{i j}\right)$ such that

$$
\begin{aligned}
2 \mathfrak{A}_{i j}\left(\Gamma_{i j}^{n}\right)= & \int_{\widehat{\mathrm{R}}_{i j}} \sqrt{1+\left|\nabla \widehat{m}_{i j}^{n}\right|^{2}} \mathrm{~d} s \mathrm{~d} t \\
= & \min \left\{\int_{\widehat{\mathrm{R}}_{i j}} \sqrt{1+|D f|^{2}}+\int_{\partial \widehat{\mathrm{R}}_{i j}}\left|f-\varphi_{i j}^{n}\right| d \mathcal{H}^{1}: f \in \operatorname{BV}(B),\right. \\
& \left.f=\varphi_{i j}^{n} \text { on } B \backslash \widehat{\mathrm{R}}_{i j}\right\}, \\
2 \mathfrak{A}_{i j}\left(\Gamma_{i j}\right)= & \int_{\widehat{\mathrm{R}}_{i j}} \sqrt{1+\left|\nabla \widehat{m}_{i j}\right|^{2}} \mathrm{~d} s \mathrm{~d} t \\
= & \min \left\{\int_{\widehat{\mathrm{R}}_{i j}} \sqrt{1+|D f|^{2}}+\int_{\partial \widehat{\mathrm{R}}_{i j}}\left|f-\varphi_{i j}\right| d \mathcal{H}^{1}: f \in \mathrm{BV}(B),\right. \\
& \left.f=\varphi_{i j} \text { on } B \backslash \widehat{\mathrm{R}}_{i j}\right\},
\end{aligned}
$$

where we recall that $\widehat{\mathrm{R}}_{i j}$ is the double rectangle defined in (3.5) and $\varphi_{i j}, \varphi_{i j}^{n}$ are extended on a disk $B$ containing $\widehat{\mathrm{R}}_{i j}$ as in Sect. 3.1.

Define $\tilde{m}_{i j}^{n}$ and $\widetilde{m}_{i j}$ as

$$
\tilde{m}_{i j}^{n}:=\left\{\begin{array}{l}
\widehat{m}_{i j}^{n} \text { in } \widehat{\mathrm{R}}_{i j}, \\
\varphi_{i j} \text { in } B \backslash \widehat{\mathrm{R}}_{i j},
\end{array} \quad \tilde{m}_{i j}:=\left\{\begin{array}{l}
\widehat{m}_{i j} \text { in } \widehat{\mathrm{R}}_{i j}, \\
\varphi_{i j}^{n} \text { in } B \backslash \widehat{\mathrm{R}}_{i j},
\end{array}\right.\right.
$$


so that $\widetilde{m}_{i j}^{n}, \widetilde{m}_{i j} \in \mathrm{BV}(B)$. Since $\widetilde{m}_{i j}^{n}$ is competitor in (5.15) and $\widetilde{m}_{i j}$ is competitor in (5.14) we have, recalling also the discussion leading to (3.8),

$$
\begin{aligned}
2 \mathfrak{A}_{i j}\left(\Gamma_{i j}\right) & \leq \int_{\widehat{\mathrm{R}}_{i j}} \sqrt{1+\left|\nabla \widehat{m}_{i j}^{n}\right|^{2}} \mathrm{~d} s \mathrm{~d} t+\int_{\partial \widehat{\mathrm{R}}_{i j}}\left|\varphi_{i j}^{n}-\varphi_{i j}\right| \mathrm{d} \mathcal{H}^{1}=2 \mathfrak{A}_{i j}^{n}+\int_{\partial \widehat{\mathrm{R}}_{i j}}\left|\varphi_{i j}^{n}-\varphi_{i j}\right| \mathrm{d} \mathcal{H}^{1}, \\
2 \mathfrak{A}_{i j}\left(\Gamma_{i j}^{n}\right) & \leq \int_{\widehat{\mathrm{R}}_{i j}} \sqrt{1+\left|\nabla \widehat{m}_{i j}\right|^{2}} \mathrm{~d} s \mathrm{~d} t+\int_{\partial \widehat{\mathrm{R}}_{i j}}\left|\varphi_{i j}-\varphi_{i j}^{n}\right| \mathrm{d} \mathcal{H}^{1}=2 \mathfrak{A}_{i j}+\int_{\partial \widehat{\mathrm{R}}_{i j}}\left|\varphi_{i j}-\varphi_{i j}^{n}\right| \mathrm{d} \mathcal{H}^{1} .
\end{aligned}
$$

Thus

$$
\left|2 \mathfrak{A}_{i j}\left(\Gamma_{i j}^{n}\right)-2 \mathfrak{A}_{i j}\left(\Gamma_{i j}\right)\right| \leq \int_{\partial \widehat{\mathrm{R}}}\left|\varphi_{i j}^{n}-\varphi_{i j}\right| \mathrm{d} \mathcal{H}^{1} .
$$

Recall that $m_{i j}^{n}$ (resp. $m_{i j}$ ) is the restriction of $\widehat{m}_{i j}^{n}$ (resp. $\widehat{m}_{i j}$ ) to $\mathrm{R}_{i j}$. Hence, from (3.9), (5.1) and (5.16), formula (5.13) follows.

Corollary 5.5 We have

$$
\inf \{\mathcal{G}(\Gamma): \Gamma \in X\}=\inf \left\{\mathcal{G}(\Gamma): \Gamma \in X_{\text {Lip }}\right\} .
$$

\subsection{Compactness of the class $X$}

The aim of this section is to show that the infimum in (5.17) is attained. To do this we need the following result.

Theorem 5.6 (Compactness) Any sequence $\left\{\Gamma^{n}\right\} \subset X$ admits a subsequence converging in $X$ to some $\Gamma \in X$.

Remark 5.7 In Definition 5.1 it is required convergence of $\left\{\Gamma^{n}\right\}$ to $\Gamma$ in $L^{1}$. For this reason, if $\Gamma^{n}$ has target triple point $p_{n}$, it is not guaranteed that the point $b:=\lim _{n \rightarrow+\infty} p_{n}$ (it exists up to subsequences) still belongs to $\Gamma_{i j}$ for all $i j$, see Figs. 12 and $15 \mathrm{a}$. As a consequence, if $\left\{\Gamma^{n}\right\}$ converges to $\Gamma$ it is not true, in general, that $p_{n} \rightarrow p$, where $p$ is the target triple point of $\Gamma$.

Proof Let $\left\{\Gamma^{n}\right\} \subset X$ and $\varphi_{i j}^{n}=\varphi_{i j}\left(\Gamma_{i j}^{n}\right), i j \in\{12,23,31\}$. From Proposition 5.3 it follows that $\left\{\varphi_{i j}^{n}\right\}$ is uniformly bounded in $\mathrm{BV}\left(\left[0, \ell_{i j}\right]\right)$ for any $i j \in\{12,23,31\}$. Thus, up to a not relabeled subsequence, there exists $\varphi_{i j} \in \mathrm{BV}\left(\left[0, \ell_{i j}\right]\right)$ such that

$$
\begin{aligned}
& \varphi_{i j}^{n} \rightarrow \varphi_{i j} \text { in } L^{1}\left(\left(0, \ell_{i j}\right)\right) \text { and pointwise a.e., } \\
& \left(\varphi_{i j}^{n}\right)^{\prime} \rightarrow \varphi_{i j}^{\prime} \quad \text { weakly* as measures. }
\end{aligned}
$$

We shall adopt our usual convention

$$
\varphi_{i j}^{n}\left(0_{-}\right)=\varphi_{i j}\left(0_{-}\right)=\varphi_{i j}^{n}\left(\ell_{i j_{+}}\right)=\varphi_{i j}\left(\ell_{i j_{+}}\right)=0, \quad \varphi_{i j}^{n}=\varphi_{i j_{+}}^{n}, \quad \varphi_{i j}=\varphi_{i j_{+}}, \quad \varphi=\varphi_{+} .
$$

Denote by $\Gamma_{i j} \subset \mathbb{R}^{2}$ the limit graph over (the closed segment) $\overline{\alpha_{i} \alpha_{j}}$ that we identify with the generalized graph of $\varphi_{i j}$ over $\left[0, \ell_{i j}\right]$. Since T is closed and convex we have $\Gamma_{i j} \subset T$; moreover, by construction, $\alpha_{i}$ and $\alpha_{j}$ are the endpoints of $\Gamma_{i j}$. Notice that if we assume that $\mathrm{T}$ is acute, this excludes the presence of vertical parts over its vertices.

It remains to prove that the three obtained curves $\Gamma_{i j}, i j \in\{12,23,31\}$, form a BV connection; in particular that they intersect mutually in a unique well-defined point. 
We claim that there exists a unique $p \in \bigcap_{i j} \Gamma_{i j}$ that divides each $\Gamma_{i j}$ into two curves $\Gamma_{i j}^{l}$ and $\Gamma_{i j}^{r}$ such that

$$
\Gamma_{i j}^{l}=\Gamma_{k i}^{r}, \quad i j, k i \in\{12,23,31\}, i j \neq k i .
$$

Let us denote by $\tilde{\varphi}_{i j}^{n}$ the extension to $\mathbb{R}$ of the function $\varphi_{i j}^{n}$ vanishing in $(-\infty, 0) \cup\left(\ell_{i j},+\infty\right)$. Similarly $\tilde{\varphi}_{i j}$ is the extension of $\varphi_{i j}$ vanishing in $(-\infty, 0) \cup\left(\ell_{i j},+\infty\right)$. Consider the sequence $\left\{\llbracket S \mathcal{G}_{\tilde{\varphi}_{i j}^{n}} \rrbracket\right\}_{n} \subset \mathcal{D}_{2}\left(\mathbb{R}^{2}\right)$ of 2-currents regarded in $\mathbb{R}^{2}$ and the 2-current $\llbracket S \mathcal{G}_{\tilde{\varphi}_{i j}} \rrbracket$. Their boundaries are the currents carried by the graphs of $\tilde{\varphi}_{i j}^{n}$ and $\tilde{\varphi}_{i j}$, respectively, as defined in Theorem 2.1. The 1-currents carried by the graph of $\varphi_{i j}^{n}$ and $\varphi_{i j}$, by convention (5.20), coincide with the restrictions of $\partial \llbracket S \mathcal{G}_{\tilde{\varphi}_{i j}^{n}} \rrbracket$ and $\partial \llbracket S G_{\tilde{\varphi}_{i j}} \rrbracket$ to the closed set $\left[0, \ell_{i j}\right] \times \mathbb{R}$. Namely, if we denote by

$$
\llbracket \Gamma_{i j}^{n} \rrbracket:=\partial \llbracket S \mathcal{G}_{\tilde{\varphi}_{i j}^{n}} \rrbracket\left\llcorner\left[0, \ell_{i j}\right] \times \mathbb{R}, \quad \llbracket \Gamma_{i j} \rrbracket:=\partial \llbracket S G_{\tilde{\varphi}_{i j}} \rrbracket\left\llcorner\left[0, \ell_{i j}\right] \times \mathbb{R},\right.\right.
$$

then

$$
\llbracket \Gamma_{i j}^{n} \rrbracket=\partial \llbracket S \mathcal{G}_{\tilde{\varphi}_{i j}^{n}} \rrbracket-\mathcal{L}_{i j} \quad \text { and } \quad \llbracket \Gamma_{i j} \rrbracket=\partial \llbracket S G_{\tilde{\varphi}_{i j}} \rrbracket-\mathcal{L}_{i j},
$$

where $\mathcal{L}_{i j}$ is the 1 -current given by integration over the two halflines $(-\infty, 0) \times\{0\} \cup$ $\left(\ell_{i j},+\infty\right) \times\{0\}$. The curves $\Gamma_{i j}^{n}$ and $\Gamma_{i j}$ coincide with the support of $\llbracket \Gamma_{i j}^{n} \rrbracket$ and $\llbracket \Gamma_{i j} \rrbracket$, respectively.

We now prove our claim in three steps.

Step 1 The currents $\llbracket \Gamma_{i j}^{n} \rrbracket$ converge (up to a not relabeled subsequence) weakly in the sense of currents to $\llbracket \Gamma_{i j} \rrbracket$, i.e.,

$$
\llbracket \Gamma_{i j}^{n} \rrbracket(\omega) \rightarrow \llbracket \Gamma_{i j} \rrbracket(\omega) \quad \forall \omega \in \mathcal{D}^{1}\left(\mathbb{R}^{2}\right) .
$$

Moreover

$$
\mathcal{H}^{1}\left(\Gamma_{i j}\right) \leq c,
$$

where $c>0$ is the constant in (5.9).

Indeed, thanks to (5.18), the characteristic functions $\chi_{S \mathcal{G}_{\varphi_{i j}^{n}}}$ converge to $\chi_{S \mathcal{G}_{\varphi_{i j}}}$ in $L_{\text {loc }}^{1}\left(\mathbb{R}^{2}\right)$, hence

$$
\llbracket S \mathcal{G}_{\tilde{\varphi}_{i j}^{n}} \rrbracket \rightarrow \llbracket S \mathcal{G}_{\tilde{\varphi}_{i j}} \rrbracket \text { weakly as currents, }
$$

since

$$
\int_{S \mathcal{G}_{\tilde{\varphi}_{i j}^{n}}^{n}} \hat{\omega}(s, t) \mathrm{d} s \mathrm{~d} t \rightarrow \int_{S \mathcal{G}_{\tilde{\varphi}_{i j}}} \hat{\omega}(s, t) \mathrm{d} s \mathrm{~d} t \quad \forall \hat{\omega} \in \mathcal{D}^{2}\left(\mathbb{R}^{2}\right)
$$

This implies

$$
\partial \llbracket S \mathcal{G}_{\tilde{\varphi}_{i j}^{n}} \rrbracket \rightarrow \partial \llbracket S \mathcal{G}_{\tilde{\varphi}_{i j}} \rrbracket \text { weakly in the sense of currents, }
$$

and (5.22) follows from (5.21).

Finally (5.23) follows from Lemma 5.3 and the weak lower semicontinuity of the mass of currents, and the proof of step 1 is concluded.

It is not restrictive to assume that $w_{i j}^{n}=\left|\alpha_{i}-\pi_{i j}\left(p_{n}\right)\right|$ is a point of continuity of $\varphi_{i j}^{n}$ for all $n \in \mathbb{N}$ and all $i j \in\{12,23,31\}$. Indeed given a sequence $\left\{\Gamma^{n}\right\} \subset X$ converging to $\Gamma$, from Lemma 5.2 for all $n$ we can assign a sequence $\left\{\Gamma^{m, n}\right\} \subset X_{\text {Lip }}$ such that $\Gamma^{m, n} \rightarrow \Gamma^{n}$ as $m \rightarrow+\infty$. Thus by a diagonal argument, we find a sequence $\left\{\Gamma^{m(n), n}\right\} \subset X_{\text {Lip }}$ which 
tends to $\Gamma$ and satisfies the above requirement (we can also assume that $\Gamma^{n}$ is Lipschitz, but this will not be needed in the proof).

Without loss of generality (up to a not relabeled subsequence), we may further assume

$$
p_{n} \rightarrow b \in \mathrm{T}
$$

$\left\{w_{i j}^{n}\right\}$ is a monotone sequence, and

$$
w_{i j}^{n} \rightarrow w_{i j}:=\left|\alpha_{i}-\pi_{i j}(b)\right|, \quad i j \in\{12,23,31\} .
$$

Before passing to the second step, it is convenient to divide the target triangle $\mathrm{T}$ into various regions.

Assume first that $\mathrm{T}$ is acute. The point $b$, together with the heights

$$
h_{i j}:=\overline{\pi_{i j}(b) b}, \quad i j \in\{12,23,31\},
$$

divides $\mathrm{T}$ into three regions $\mathcal{P}_{i}, i \in\{1,2,3\}$, as shown in Fig. 11a; precisely, if $\overline{\mathcal{P}}_{i}$ denotes the closed region enclosed by $h_{i j}, h_{k i}, \overline{\alpha_{i} \pi_{i j}(b)}$ and $\overline{\alpha_{i} \pi_{k i}(b)}$, then $\mathcal{P}_{i}$ is defined by

$$
\mathcal{P}_{i}:=\overline{\mathcal{P}}_{i} \backslash\left(h_{i j} \cup h_{k i}\right), \quad i=1,2,3 .
$$

Similarly we define $h_{i j}^{n}$ and $\mathcal{P}_{i}^{n}$ by replacing $b$ with $p_{n}$ in (5.24) and (5.25).

Assume now that $\mathrm{T}$ is not acute. Without loss of generality, we may assume that the angle at $\alpha_{1}$ is greater than $\frac{\pi}{2}$. The only difference here is with the definition of $\mathcal{P}_{1}^{n}$ and $\mathcal{P}_{1}$, since each $\Gamma_{i j}$ has to satisfy the graphicality condition with respect to $\overline{\alpha_{i} \alpha_{j}}$; hence we define $\overline{\mathcal{P}_{1}}$ as the closed quadrilateral bounded by $h_{12}, h_{31}, n_{12}$ and $n_{31}$, where $n_{12}$ and $n_{31}$ are the normals to $\overline{\alpha_{1} \alpha_{2}}$ and $\overline{\alpha_{3} \alpha_{1}}$, respectively, passing through $\alpha_{1}$ (see Fig. 11b). Similarly we define $\overline{\mathcal{P}_{1}^{n}}$. Finally we set $\mathcal{P}_{1}:=\overline{\mathcal{P}_{1}} \backslash\left(h_{12} \cup h_{31}\right)$ and $\mathcal{P}_{1}^{n}:=\overline{\mathcal{P}_{1}^{n}} \backslash\left(h_{12} \cup h_{31}\right)$.

Step 2 We will prove that we can decompose $\Gamma_{12} \cup \Gamma_{23} \cup \Gamma_{31}$ as three currents meeting at a point $b$.

It is easy to see that the sets $\overline{\mathcal{P}_{i}^{n}}$ are converging to $\overline{\mathcal{P}}_{i}$ with respect to the Hausdorff distance. It is not true in general that $\left\{\Gamma_{i j}^{n}\right\}$ is converging to $\Gamma_{i j}$ in the Hausdorff distance (see Fig. 12); however, since

$$
\Gamma_{i}^{n}=\Gamma_{i j}^{n} \cap \overline{\mathcal{P}_{i}^{n}}=\Gamma_{k i}^{n} \cap \overline{\mathcal{P}_{i}^{n}}, \quad \Gamma_{i}^{n} \subset \overline{\mathcal{P}}_{i}^{n},
$$

for all $i j, k i \in\{12,23,31\}, i j \neq k i$, it is readily seen that

$$
\begin{aligned}
& \Gamma_{i j} \subset \mathrm{T} \backslash \mathcal{P}_{k}, \\
& \llbracket \Gamma_{i j} \rrbracket=\llbracket \Gamma_{i j} \rrbracket\left\llcorner\mathcal{P}_{i}+\llbracket \Gamma_{i j} \rrbracket\left\llcorner\mathcal{P}_{j}+\llbracket \Gamma_{i j} \rrbracket\left\llcorner h_{k i}+\llbracket \Gamma_{i j} \rrbracket\left\llcorner h_{i j}+\llbracket \Gamma_{i j} \rrbracket\left\llcorner h_{j k} .\right.\right.\right.\right.\right.
\end{aligned}
$$

For $i \in\{1,2,3\}$, the integral 1-current $\llbracket \Gamma_{i}^{n} \rrbracket=\llbracket \Gamma_{i j}^{n} \rrbracket\left\llcorner\left[0, w_{i j}\right] \times \mathbb{R}\right.$ has boundary $\delta_{p_{n}}-\delta_{\alpha_{i}}$ in $\mathbb{R}^{2}$. By the compactness theorem for integral currents [10, Theorem 2, p.141] there exists an integral current $\mathcal{T}_{i} \in \mathcal{D}_{1}\left(\mathbb{R}^{2}\right), i=1,2,3$, such that, up to a not relabeled subsequence,

$$
\llbracket \Gamma_{i}^{n} \rrbracket(\omega) \rightarrow \mathcal{T}_{i}(\omega) \quad \forall \omega \in \mathcal{D}^{1}\left(\mathbb{R}^{2}\right) .
$$

Clearly

$$
\partial \mathcal{T}_{i}=\delta_{b}-\delta_{\alpha_{i}}
$$

From (5.26) and the convergence of $\overline{\mathcal{P}_{i}^{n}}$ to $\overline{\mathcal{P}_{i}}$ in the Hausdorff distance, we infer

$$
\text { spt } \mathcal{T}_{i} \subset \overline{\mathcal{P}_{i}}, \text { hence } \mathcal{T}_{i}=\mathcal{T}_{i}\left\llcorner\mathcal{P}_{i}+\mathcal{T}_{i}\left\llcorner h_{i j}+\mathcal{T}_{i}\left\llcorner h_{k i},\right.\right.\right.
$$




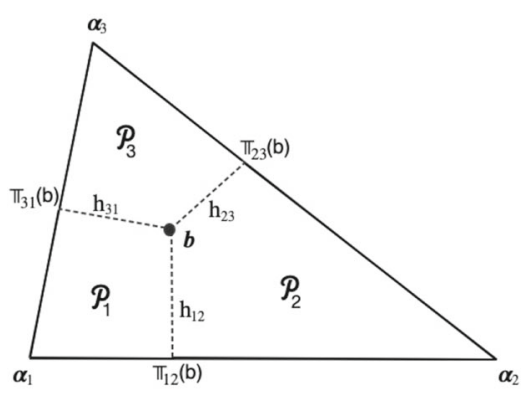

(a)

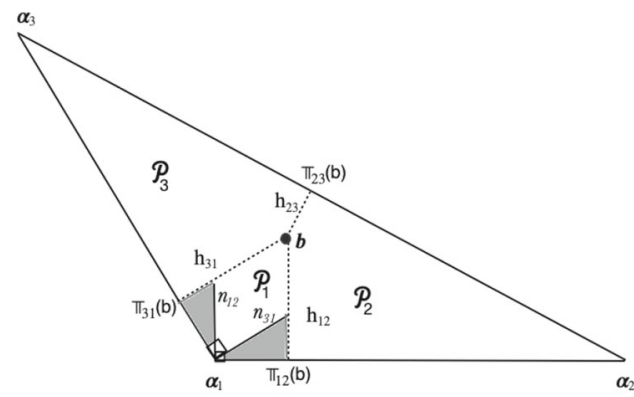

(b)

Fig. 11 a Partitions of T into $\mathcal{P}_{1}, \mathcal{P}_{2}, \mathcal{P}_{3}$ and three segments and $\mathbf{b}$ partitions of $\mathrm{T}$ minus the two gray triangles into $\mathcal{P}_{1}, \mathcal{P}_{2}, \mathcal{P}_{3}$ and three segments for a non-acute triangle

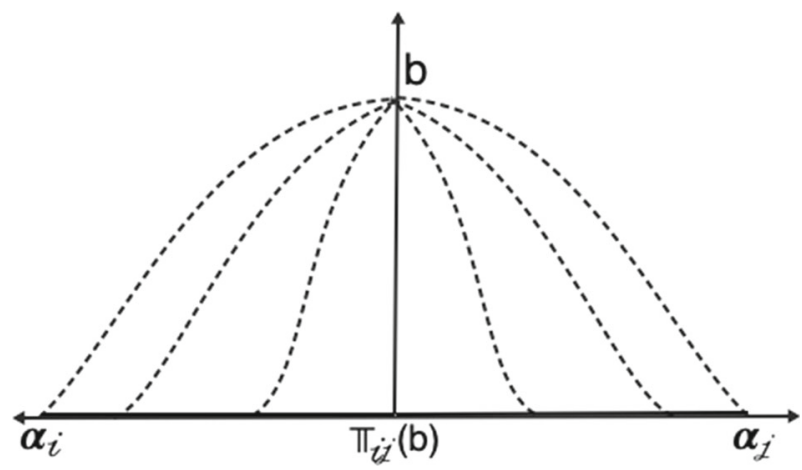

Fig. 12 In dots a sequence of graphs $\llbracket \Gamma_{i j}^{n} \rrbracket$ of functions that pass through a fixed point $b \in T$. In bold the graph of the limit function (the horizontal segment) $\llbracket \Gamma_{i j} \rrbracket$. The limit in the sense of currents of the left branches of the sequence $\left\{\Gamma_{i}^{n}\right\}$ is $\overline{\alpha_{i} \pi_{i j}(b)} \cup \overline{\pi_{i j}(b) b}$ while the limit of the right branches $\left\{\Gamma_{j}^{n}\right\}$ is $\overline{\pi_{i j}(b) b} \cup \overline{\pi_{i j}(b) \alpha_{j}}$

where $i j, k i \in\{12,23,31\}$. Note that $\mathcal{T}_{i}$ is not necessarily equal to $\llbracket \Gamma_{i j} \rrbracket\left\llcorner\overline{\mathcal{P}_{i}}\right.$, due to a possible cancelation of a vertical part over $\pi_{i j}(b), i j \in\{12,23,31\}$ (that is, on $h_{i j}$ ), see Fig. 12. However, from $\llbracket \Gamma_{i j}^{n} \rrbracket=\llbracket \Gamma_{i}^{n} \rrbracket+\llbracket \Gamma_{j}^{n} \rrbracket$ and (5.28) we have

$$
\llbracket \Gamma_{i j} \rrbracket=\mathcal{T}_{i}-\mathcal{T}_{j}, \quad i j \in\{12,23,31\},
$$

as currents in $\mathbb{R}^{2}$. Notice that $\mathcal{T}_{i}$ and $\mathcal{T}_{j}$ have multiplicity one, and in (5.31) they contribute with opposite orientation. This allows, if necessary, to identify $\mathcal{T}_{i}, i=1,2,3$, with its support. Note also that $\mathcal{T}_{i}$ may have vertical part over $\alpha_{i}$, see Fig. 13.

Now, since $\llbracket \Gamma_{i}^{n} \rrbracket$ is Cartesian with respect to both the edges $\overline{\alpha_{i} \alpha_{j}}$ and $\overline{\alpha_{k} \alpha_{i}}$, from (5.28) it follows that $\mathcal{T}_{i}\left\llcorner\mathcal{P}_{i}\right.$ is part of two generalized graphs over the same edges, i.e.,

$$
\mathcal{T}_{i}\left\llcorner\mathcal{P}_{i}=\llbracket \Gamma_{i j} \rrbracket\left\llcorner\mathcal{P}_{i}=-\llbracket \Gamma_{k i} \rrbracket\left\llcorner\mathcal{P}_{i} .\right.\right.\right.
$$

Moreover, we infer that $\mathcal{T}_{i}$ cannot have vertical part over $h_{i j}$ and $h_{k i}$ at the same time; in other words once the current $\mathcal{T}_{i}$ touches one of the heights $h_{i j}$ or $h_{k i}$ it stays there until it reaches $b$, and $\mathcal{T}_{i}$ cannot have a nonempty support in more than one height, see Figs. 14b and $15 \mathrm{a}, \mathrm{b}$. We conclude the following statement: 


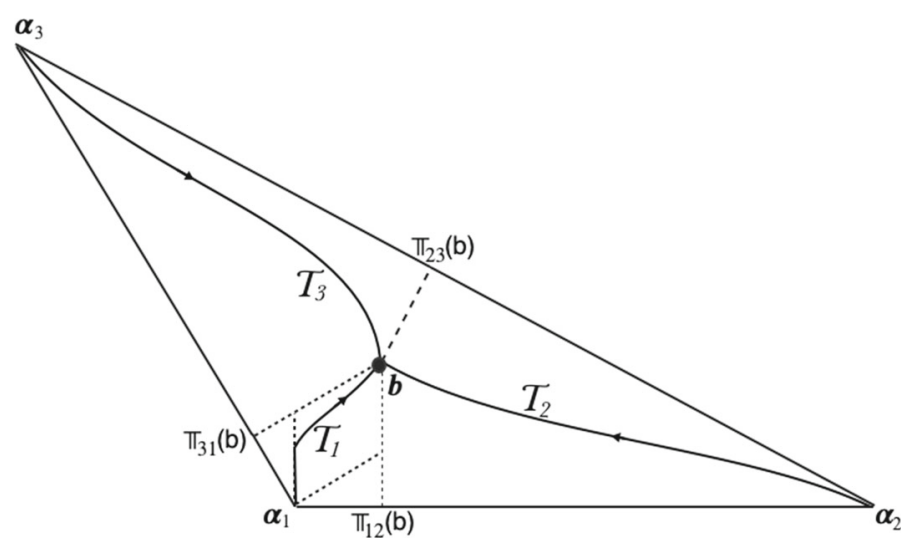

Fig. $13 \mathcal{T}_{1}$ has a vertical part over $\alpha_{1}$

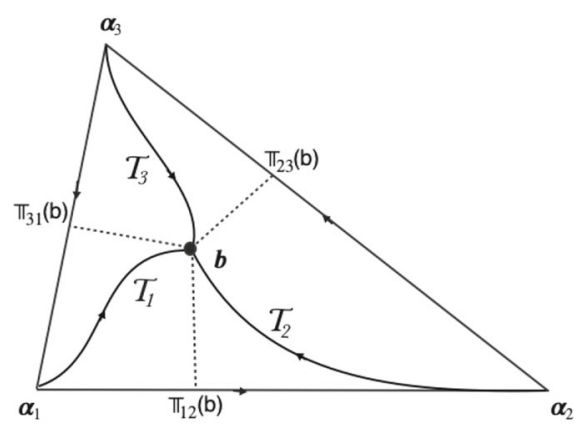

(a)

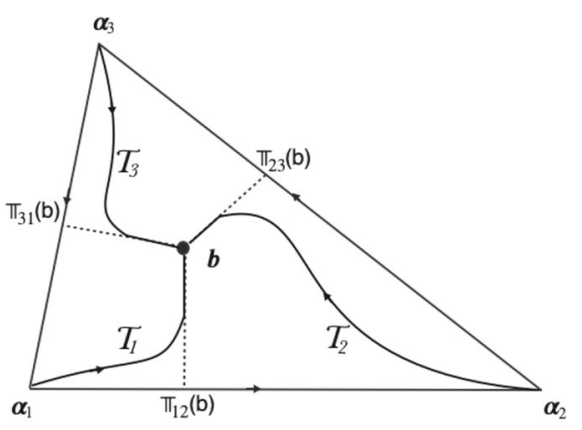

(b)

Fig. 14 Case (i) of step 3 in the proof of Theorem 5.6

(A) The supports of the three currents $\mathcal{T}_{i}, i=1,2,3$, have as common point $b$. Moreover, if there are $i \neq j$ such that the supports of $\mathcal{T}_{i}$ and $\mathcal{T}_{j}$ intersect in a point different from $b$, then this intersection occurs on the mutual height $h_{i j}$. Finally, if the supports of $\mathcal{T}_{i}$ and $\mathcal{T}_{j}$ intersect on $h_{i j}$ outside $b$, then they intersect on a closed segment and the intersection of the supports of $\mathcal{T}_{i}$ and $\mathcal{T}_{j}$ with $\mathcal{T}_{k}$ is only the point $b$.

Step 3 To conclude the proof of our claim we now analyze the possible cases arising from (A).

Case (i) Assume that the three supports of the currents $\mathcal{T}_{i}, i=1,2,3$, intersect only at the point $b$. This includes the case

$$
\mathcal{T}_{i}\left\llcorner h_{i j}=\mathcal{T}_{j}\left\llcorner h_{i j}=0 \text { for all } i j \in\{12,23,31\},\right.\right.
$$

as in Fig. 14a. But it may also happen that $\mathcal{T}_{i}$ has vertical part over $h_{i j}$, provided that $\mathcal{T}_{j}$ does not have vertical part over the same height (see for instance Fig. 14b). In any case we may set

$$
p:=b, \quad \Gamma_{i j}^{l}=\mathcal{T}_{i}, \quad \Gamma_{i j}^{r}:=-\mathcal{T}_{j}, \quad i j \in\{12,23,31\},
$$

where we have identified the currents $\mathcal{T}_{i}$ with their supports. By (5.29) and (5.32), the claim is achieved. 


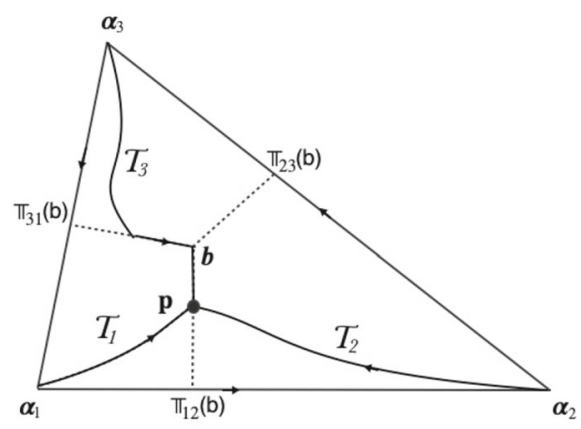

(a) $\mathcal{T}_{1}$ and $\mathcal{T}_{2}$ coincide only on $\overline{b p}$.

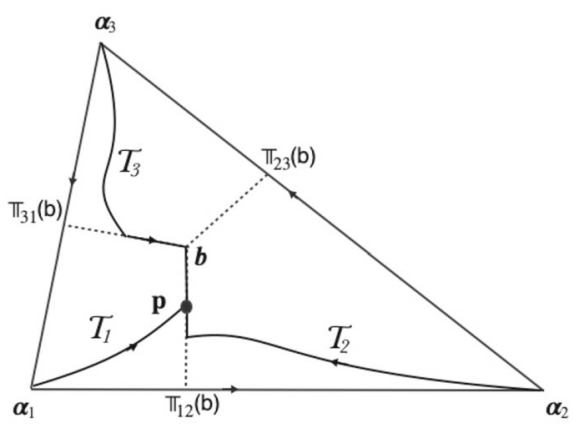

(b) $\mathcal{T}_{1}$ and $\mathcal{T}_{2}$ overlap on $\overline{b p}$ and $\mathcal{T}_{1}\left\llcorner\overline{b p} \subset \mathcal{T}_{2}\llcorner\overline{b p}\right.$.

Fig. 15 Case (ii) of step 3 in the proof of Theorem 5.6

Case (ii) The second case to be discussed is the one considering possible overlapping of the support of the currents $\mathcal{T}_{i}$. By condition (A) such overlapping, giving rise to cancelations, can occur only on one height $h_{i j}$. Hence, assume there exists one (and only one) $i j \in\{12,23,31\}$ such that

$$
\mathcal{T}_{i}\left\llcornerh _ { i j } \neq 0 \text { and } \mathcal { T } _ { j } \left\llcorner h_{i j} \neq 0\right.\right. \text {. }
$$

Thus we have $\mathcal{T}_{i}\left\llcorner h_{k i}=0\right.$ and $\mathcal{T}_{j}\left\llcorner h_{j k}=0\right.$.

First assume that $\llbracket \Gamma_{i j} \rrbracket\left\llcorner h_{i j}=0\right.$, i.e., $\varphi_{i j}$ is continuous at $w_{i j}$. Then $\mathcal{T}_{i}\left\llcorner h_{i j}=\mathcal{T}_{j}\left\llcorner h_{i j}\right.\right.$, see Fig. 15a. We set, identifying $\mathcal{T}_{i}$ with its support,

$$
\begin{array}{ll}
p:=\varphi_{i j}\left(w_{i j}\right), & \\
\Gamma_{i j}^{l}:=\mathcal{T}_{i}\left\llcorner\mathcal{P}_{i},\right. & \Gamma_{i j}^{r}:=\mathcal{T}_{j}\left\llcorner\mathcal{P}_{j},\right. \\
\Gamma_{j k}^{l}:=\mathcal{T}_{j}\left\llcorner\mathcal{P}_{i},\right. & \Gamma_{j k}^{r}:=\mathcal{T}_{k} \cup \mathcal{T}_{j}\left\llcorner h_{i j},\right. \\
\Gamma_{k i}^{l}:=\mathcal{T}_{k} \cup \mathcal{T}_{i}\left\llcorner h_{i j},\right. & \Gamma_{k i}^{r}:=\mathcal{T}_{i}\left\llcorner\mathcal{P}_{i} .\right.
\end{array}
$$

One checks that the connection built above is a BV graph type connection, addressing the claim.

Now assume that

$$
\llbracket \Gamma_{i j} \rrbracket\left\llcorner h_{i j} \neq 0,\right.
$$

i.e., $\varphi_{i j}$ jumps at $w_{i j}$. Thus either spt $\llbracket \Gamma_{i j} \rrbracket\left\llcorner h_{i j} \subseteq\right.$ spt $\mathcal{T}_{i}\left\llcorner h_{i j}\right.$ or spt $\llbracket \Gamma_{i j} \rrbracket\left\llcorner h_{i j} \subseteq\right.$ spt $\mathcal{T}_{j}\left\llcorner h_{i j}\right.$. Without loss of generality we may assume that spt $\llbracket \Gamma_{i j} \rrbracket\left\llcorner h_{i j} \subseteq\right.$ spt $\mathcal{T}_{i}\left\llcorner h_{i j}\right.$, hence $\mathcal{T}_{i}\left\llcorner\left(h_{i j} \backslash\right.\right.$ spt $\left.\llbracket \Gamma_{i j} \rrbracket\right)=-\mathcal{T}_{j}\left\llcorner h_{i j} \neq 0\right.$ (note that spt $\llbracket \Gamma_{i j} \rrbracket\left\llcorner h_{i j}=\left\{t \varphi_{i j}\left(w_{i j+}\right)+\right.\right.$ $\left.\left.(1-t) \varphi_{i j}\left(w_{i j-}\right): t \in[0,1]\right\}\right)$. We set

$$
\begin{array}{ll}
p:=\varphi_{i j}\left(w_{i j}\right)=\varphi_{i j+}\left(w_{i j}\right), & \\
\Gamma_{i j}^{l}:=\mathcal{T}_{i}\left\llcorner\mathcal{P}_{i} \cup\left(h_{i j} \cap \operatorname{spt} \llbracket \Gamma_{i j} \rrbracket\right),\right. & \Gamma_{i j}^{r}:=\mathcal{T}_{j}\left\llcorner\mathcal{P}_{j},\right. \\
\Gamma_{j k}^{l}:=\mathcal{T}_{j}\left\llcorner\mathcal{P}_{i},\right. & \Gamma_{j k}^{r}:=\mathcal{T}_{k} \cup \mathcal{T}_{j}\left\llcorner h_{i j},\right. \\
\Gamma_{k i}^{l}:=\mathcal{T}_{k} \cup \mathcal{T}_{j}\left\llcorner h_{i j},\right. & \Gamma_{k i}^{r}:=\mathcal{T}_{i}\left\llcorner\mathcal{P}_{i} \cup\left(h_{i j} \cap \operatorname{spt} \llbracket \Gamma_{i j}^{n} \rrbracket\right),\right.
\end{array}
$$

see Fig. 15b. Also in this case the conclusion follows. 
In the end, it is enough to define

$$
\Gamma:=\left(\Gamma_{1}, \Gamma_{2}, \Gamma_{3}\right), \quad \Gamma_{i}:=\Gamma_{i j}^{l}=\Gamma_{k i}^{r}, \quad i=1,2,3 .
$$

From the compactness of the space of BV connections, combining with Proposition 5.4, we see that the infimum in (4.1) is attained. As a consequence, we can conclude the proof of Theorem 1.1.

\section{Corollary 5.8 We have}

$$
\mathcal{A}(u, D) \leq|D|+\inf \left\{\mathcal{G}(\Gamma): \Gamma \in X_{\mathrm{Lip}}\right\}=|D|+\min \{\mathcal{G}(\Gamma): \Gamma \in X\} .
$$

Acknowledgements The present paper benefits from the support of the GNAMPA (Gruppo Nazionale per l'Analisi Matematica, la Probabilità e le loro Applicazioni) of INdAM (Istituto Nazionale di Alta Matematica).

\section{References}

1. Acerbi, E., Dal Maso, G.: New lower semicontinuity results for polyconvex integrals. Calc. Var. Partial Differ. Equ. 2, 329-371 (1994)

2. Ambrosio, L.: Geometric evolution problems, distance function and viscosity solutions. In: Buttazzo, G., Marino, A., Murthy, M.K.V. (eds.) Calculus of Variations and Partial Differential Equations (Pisa, 1996), pp. 5-93. Springer, Berlin (2000)

3. Ambrosio, L., Fusco, N., Pallara, D.: Functions of Bounded Variation and Free Discontinuity Problems. Mathematical Monographs. Oxford University Press, Oxford (2000)

4. Bellettini, G., Paolini, M.: On the area of the graph of a singular map from the plane to the plane taking three values. Adv. Calc. Var. 3, 371-386 (2010)

5. Bellettini, G., Paolini, M., Tealdi, L.: On the area of the graph of a piecewise smooth map from the plane to the plane with a curve discontinuity. ESAIM Control Optim. Calc. Var. 22, 29-63 (2015)

6. Bellettini, G., Paolini, M., Tealdi, L.: Semicartesian surfaces and the relaxed area of maps from the plane to the plane with a line discontinuity. Ann. Mat. Pura Appl. 195, 2131-2170 (2016)

7. Dal Maso, G.: Integral representation on $B V(\Omega)$ of $\Gamma$-limits of variational integrals. Manuscr. Math. 30, 387-416 (1980)

8. De Giorgi, E.: On the relaxation of functionals defined on cartesian manifolds. In: Buttazzo, G., Galdi, G.P., Zanghirati, L. (eds.) Developments in Partial Differential Equations and Applications in Mathematical Physics (Ferrara 1992). Plenum Press, New York (1992)

9. Fusco, N., Hutchinson, J.E.: A direct proof for lower semicontinuity of polyconvex functionals. Manuscr. Mat. 87, 30-35 (1995)

10. Giaquinta, M., Modica, G., Souček, J.: Cartesian Currents in the Calculus of Variations I. Cartesian currents. Ergebnisse der Mathematik und ihrer Grenzgebiete. 3. Folge. 37. Springer, Berlin (1998)

11. Giusti, E.: Minimal Surfaces and Functions of Bounded Variation. Birkhäuser, Boston (1984)

12. Scala, R.: Optimal estimates for the triple junction function and other surprising aspects of the area functional. Ann. Sc. Norm. Super. Pisa Cl. Sci. 20(2)

Publisher's Note Springer Nature remains neutral with regard to jurisdictional claims in published maps and institutional affiliations. 